\title{
Theoretical Effect of Yield and Burst Height of Atmospheric Explosions on Rayleigh Wave Amplitudes*
}

\author{
D. G. Harkrider, C. A. Newton $\uparrow$ and E. A. Flinn
}

(Received 1973 March 26)

\begin{abstract}
Summary
Theoretical seismograms for fundamental mode Rayleigh waves were calculated for atmospheric point sources over oceanic and over continental Earth models, as recorded at an epicentral distance of $10000 \mathrm{~km}$. Yields were uniformly distributed over the range $1 \mathrm{kT}-10 \mathrm{MT}$, for source altitudes in the range $0.3-92.0 \mathrm{~km}$. The Earth structures used were those of Gutenberg and of Anderson and Toksöz. The source models were point mass-injection and energy-injection sources at altitude, as well as a distributed pressure pulse at the surface of the Earth.

It was found that: (1) as far as Rayleigh wave excitation is concerned, the mass-injection and energy-injection sources are equivalent; (2) for low altitudes the Rayleigh wave excitation is independent of source type, but at intermediate altitudes the surface overpressure source predicts greater amplitudes than the other two source models; (3) for most altitudes, the energy coupling from the atmosphere into Rayleigh waves is more efficient for the continental Earth structure than for the oceanic structure; (4) Rayleigh wave amplitude is more sensitive to yield than to burst height (5) dependence of Rayleigh wave amplitude is less than the cube root relation for low-yield explosions at intermediate altitudes but greater for high-yield explosions at near-surface altitudes; (6) spectral splitting ratios do not show a systematic variation with yield and burst height.
\end{abstract}

\section{Symbols not defined in text}

g gravitational acceleration;

$\sigma_{2} \quad$ isothermal Brunt frequency;

$\alpha \quad$ sound velocity;

$\omega \quad$ angular frequency;

$P_{0} \quad$ ambient pressure at sea level;

$P_{\mathrm{s}}(D)$ ambient pressure at source height;

$p_{a_{0}} \quad$ peak overpressure of $1 \mathrm{kT}$ source at distance $a_{0}$;

* Contribution No. 2272, Division of Geological and Planetary Sciences, California Institute of Technology, Pasadena, California.

$\dagger$ Present address: Seismological Laboratory, California Institute of Technology, Pasadena, California. 
c $\quad$ phase velocity $(\omega / k)$;

$k$ wave number;

$T_{a \mathrm{~s}} \quad$ positive phase duration;

$a_{s} \quad$ scaling distance;

$\rho_{0} \quad$ ambient density at sea level;

$\rho_{\mathrm{s}}(D)$ ambient density at source height;

$w_{0} \quad$ vertical surface displacement;

$\gamma \quad$ attenuation coefficient;

$a_{\mathrm{e}} \quad$ Earth radius;

$\theta_{\mathrm{e}} \quad$ epicentral distance in degrees;

$\mathscr{A}_{\mathrm{R}} \quad$ Rayleigh wave medium response, continental structure;

$\mathscr{A}$ e Rayleigh wave medium response, Earth/atmosphere structure;

$\mathscr{A}_{\sigma} \quad$ Rayleigh wave medium response, oceanic structure.

\section{Introduction}

In this paper we calculate Rayleigh wave excitation at teleseismic distances for a variety of source models, Earth structures, source yields, and burst heights, and study the variation of the Rayleigh wave spectrum and waveform with these parameters.

We used Harkrider's (1964a) computer code for calculating the far-field modal excitation of acoustic-gravity waves in an atmosphere consisting of isothermal layers overlying a rigid half space, and a similar code (Harkrider 1964b) for calculating Rayleigh wave propagation in a layered solid/liquid half space underlying a vacuum. These two codes have been merged in order to model the coupling of atmospheric and seismic wave propagation and to calculate the generation of Rayleigh waves from a point source in the atmosphere, and the latter code was also used to model a pressure source distributed on the solid free surface.

It was found that the loading of the atmosphere on the solid Earth affected the Rayleigh wave characteristics by only about 0.01 per cent, i.e. the ratio of atmospheric to solid Earth density.

Previous attempts to predict Rayleigh wave excitation by atmospheric sources were either oversimplified or else heuristic and ad hoc in nature. Toksöz \& BenMenahem (1964) assumed a point source in a homogeneous liquid half space overlying a homogeneous solid half space; they made use of an equation of Cagniard (1962) and predicted that the effect of burst height on Rayleigh wave excitation should be negligible in this situation. Nickel \& Whitaker (1971) used an ingenious synthesis technique to approximate a layered solid Earth model. We discuss below the validity of the assumptions involved in their technique.

Harkrider \& Flinn (1970) described the formulation for a point mass-injection source within one layer of the atmosphere. In the work reported here we modified the codes Harkrider \& Flinn used to include Pierce's (1968) energy-injection source as well as a distributed surface overpressure source.

The solutions to an expanding ring and an expanding disk surface load on an isotropic half space have been obtained by Gakenheimer \& Miklowitz (1969), and Gakenheimer $(1969,1971)$, among others. Rayleigh and body waves due to an atmospheric explosion modelled by an equivalent moving normal force applied to a 
multilayered half space, have been formulated by Hudson (1969) and computed for an equivalent stationary point source at the free surface by Douglas, Hudson \& Blamey (1972).

The codes used in the present work are described by Kerr (1971). Source scaling was done by standard methods (Harkrider \& Flinn 1970). Two Earth models were used in the present work: the Gutenberg continental model (which has a low-velocity zone; see Ben-Menahem \& Harkrider 1964, p. 2610) and the Anderson-Toksöz oceanic model (Harkrider \& Anderson 1966, p. 2970). Earth and atmospheric layering characteristics were as usual assumed to be invariant between source and receiver.

Calculations were made for a variety of yields and burst heights for both Earth models and for all three source models. Waveforms and spectra were calculated for yields of $1 \mathrm{kT}-10 \mathrm{MT}$ and burst heights of $0.3-80 \mathrm{~km}$.

\section{Theoretical numerical methods}

\section{Energy injection source in the atmosphere}

The formulation for Rayleigh waves generated by an explosion in a gravitating atmosphere coupled to the solid Earth by a mass injection source was described by Harkrider \& Flinn (1970). We have modified our calculations by using the Pierce energy injection source (Pierce 1968; Pierce, Posey \& Iliff 1970) which was used by them to calculate acoustic-gravity waves from an explosion in the atmosphere.

For an energy source at $(r, z)=(0, D)(z$ positive upward) in an infinite isothermal gravitating ideal gas, the outgoing time-transformed parcel pressure $\left\langle p_{p_{s}}\right\rangle$ can be written in integral form as

$\left\langle p_{p_{s}}\right\rangle=2 \pi i \omega G_{\mathrm{E}} \exp [-\lambda(z-D)] \int_{0}^{\infty}\left(1-\frac{g^{2} k^{2}}{\omega^{4}}\right) \cdot \frac{\exp (-i \bar{v}|z-D|)}{i \bar{\nu}} J_{0}(k r) k d k$

where

and

$$
\begin{gathered}
\lambda=\gamma g / 2 \alpha^{2} \\
\bar{v}^{2}=(\omega / \alpha)^{2}-h_{2} k^{2}-\lambda^{2} \\
h_{2}=1-\sigma_{2}{ }^{2} / \omega^{2}
\end{gathered}
$$

$$
\begin{gathered}
G_{\mathrm{E}}=\frac{a_{\mathrm{s}} p_{\text {as }}}{2 \pi} \frac{\exp \left[-i \phi_{\mathrm{e}}\right]}{\left(b_{\mathrm{s}}^{2}+\omega^{2}\right)} \\
p_{\mathrm{as}}=p_{\mathrm{a} 0}\left[\frac{P_{\mathrm{s}}(D)}{P_{0}}\right] \\
b_{\mathrm{s}}=1 / T_{\text {as }} \\
\phi_{\mathrm{e}}=2 \tan ^{-1}\left(\omega / b_{\mathrm{s}}\right) .
\end{gathered}
$$

Following Harkrider \& Flinn (1970), we find that

and

$$
\begin{aligned}
& \Delta v_{\mathrm{s}}=4 \pi k \frac{G_{\mathrm{E}}}{\rho_{\mathrm{s}}(D)} \\
& \Delta p_{\mathrm{s}}=0
\end{aligned}
$$

$$
\begin{aligned}
{\left[w_{0}\right]_{R_{j}}=} & \left(\frac{2}{\pi}\right)^{\frac{1}{2}} \int_{-\infty}^{\infty} \frac{p_{\mathrm{as}} a_{\mathrm{s}} k_{j}^{-\frac{1}{2}}}{\left(\omega^{2}+b_{\mathrm{s}}{ }^{2}\right)} \frac{\exp [-\hat{\gamma}(\omega) r]}{\left(a_{\mathrm{e}} \sin \theta_{\mathrm{e}}\right)^{\frac{1}{2}}} \mathscr{A}_{\mathrm{e}} \\
& \times \frac{1}{\rho_{\mathrm{s}}(D)}\left[\frac{p_{p}(D)}{\dot{w}_{0}}\right] \exp \left[i\left(\omega t-k_{j} r+3 \pi / 4-\phi_{\mathrm{e}}\right)\right] d \omega
\end{aligned}
$$


where

$$
\begin{gathered}
\mathscr{A}_{\mathrm{e}}=\frac{-i[G N-L H]}{\left(\partial F_{\mathrm{e}} / \partial k\right)_{\omega}} \\
F_{\mathrm{e}}=[N K-L M]-\frac{c \tilde{A}_{21}}{\tilde{A}_{22}}[G N-L H] \\
{\left[\frac{p_{p}(D)}{\dot{w}_{0}}\right]=-\left(\tilde{A}_{\mathrm{s}}\right)_{21}+\frac{\tilde{A}_{21}}{\tilde{A}_{22}}\left(\tilde{A}_{\mathrm{s}}\right)_{22}}
\end{gathered}
$$

$\tilde{A}_{\mathrm{s}}$ is the atmosphere product matrix evaluated at the source altitude, and $\tilde{A}$ is the same product matrix evaluated at the top of the atmosphere. $k_{j}$ is the $j$ 'th root of the Rayleigh wave dispersion equation.

It should be noted that substitution of equation (2) into the source relations of Harkrider (1964b) yields the same acoustic-gravity wave overpressure relations as those of Pierce et al. (1971).

Rayleigh wave displacements from sea-level overpressures

For the excitation of Rayleigh waves by an overpressure specified at sea level, we model the Earth by a multilayered elastic half space with an optional layered gravitating liquid at the surface. We formulate the problem for an azimuthally symmetric overpressure source $\left[p_{s}(r, t)\right]$ applied to the surface of the Earth model.

The Fourier time-transformed source

$$
\left\langle p_{\mathrm{s}}(r)\right\rangle=\int_{-\infty}^{\infty}\left[p_{\mathrm{s}}(r, t)\right] \exp (-i \omega t) d t
$$

can because of symmetry be written as

$$
\left\langle p_{\mathrm{s}}(r)\right\rangle=\int_{0}^{\infty} p_{\mathrm{s}} J_{0}(k r) d k
$$

where

$$
p_{\mathrm{s}}=k \int_{0}^{\infty}\left\langle p_{\mathrm{s}}(r)\right\rangle J_{0}(k r) r d r .
$$

The twice transformed pressures and particle velocities at the free surface of the liquid layer for the oceanic model are related to those on the ocean bottom by

$$
\left[\begin{array}{c}
-\dot{w}_{\mathrm{s}} \\
p_{\mathrm{s}}
\end{array}\right]=A\left[\begin{array}{c}
-\dot{w}_{0} \\
p_{0}
\end{array}\right]
$$

or

$$
p_{\mathrm{s}}=\left[-A_{21}+\frac{p_{0}}{\dot{w}_{0}} A_{22}\right] \dot{w}_{0}
$$

where the ocean layer matrix $A$ can be obtained from Dorman (1962) or by setting the gravitational acceleration equal to zero in the atmospheric matrices of Press \& Harkrider (1962) and Harkrider \& Flinn (1970).

If we assume the separable form of equation (4), the normal stress $\sigma_{0}$ and the vertical (positive downward) displacement $w_{0}$ at the ocean-solid Earth interface are related to the elastic properties of the solid Earth by

$$
\left[\frac{\sigma_{0}}{\dot{w}_{0} / c}\right]=-\frac{[N K-L M]}{[G N-L H]}
$$


where the quantities $N, K, L, M, G$ and $H$ are defined by Harkrider (1964a, 1970). Assuming continuity of normal stress and vertical particle velocity at the liquid-solid interface we obtain by combining equations (5) and (6):

$$
w_{0}=-i \frac{p_{\mathrm{s}}}{k} \frac{1}{A_{22}} \frac{[G N-L H]}{F_{\sigma}}
$$

where

$$
\begin{gathered}
F_{\sigma}=[N K-L M]-c \frac{A_{21}}{A_{22}}[G N-L H] \\
\dot{w}_{0}=i \omega w_{0} \quad \text { and } \quad p_{0}=-\sigma_{0} .
\end{gathered}
$$

Therefore the time-transformed vertical displacement on the ocean bottom

is given by

$$
\left\langle w_{0}\right\rangle=\int_{0}^{\infty} w_{0} J_{0}(k r) d k
$$

$$
\left\langle w_{0}\right\rangle=-i \int_{0}^{\infty} \frac{p_{\mathrm{s}}}{k} \frac{1}{A_{22}} \frac{[G N-L H]}{F_{\sigma}} J_{0}(k r) d k .
$$

For the overpressures applied to the surface of the continental model, the result is similar to the oceanic model:

where

$$
\left\langle w_{0}\right\rangle=-i \int_{0}^{\infty} \frac{p_{\mathrm{s}}}{k} \frac{[G N-L H]}{F_{\mathrm{R}}} J_{0}(k r) d k
$$

$$
F_{\mathrm{R}}=[N K-L M] \text {. }
$$

This result could also be obtained by letting the thickness of the water layer approach zero in equation (8), since $A_{22} \rightarrow 1$ and $A_{21} \rightarrow 0$ in this limit.

Evaluating the residue contribution of integrals (8) and (9), we obtain the solutions for the vertical displacement spectra associated with the $j$ 'th mode Rayleigh waves. For the oceanic model the solution is

$$
\left\{w_{0}\right\}_{j}=-\frac{\pi}{k_{j}} \frac{p_{\mathrm{s}}}{A_{22}} \frac{[G N-L H]}{\left(\partial F_{\sigma} / \partial k\right)_{j}} H_{0}^{(2)}\left(k_{j} r\right)
$$

and for the continental model

$$
\left\{w_{0}\right\}_{j}=-\frac{\pi}{k_{j}} p_{\mathrm{s}} \frac{[G N-L H]}{\left(\partial F_{\mathrm{R}} / \partial k\right)_{j}} H_{0}^{(2)}\left(k_{j} r\right)
$$

where $k_{j}$ is the $j$ 'th root of the Rayleigh wave dispersion equation for the ocean:

or the continent:

$$
F_{\sigma}\left(k_{j}, \omega\right)=0
$$

$$
F_{\mathrm{R}}\left(k_{j}, \omega\right)=0 \text {. }
$$

Although we restricted the problem to azimuthal symmetry, the solutions for more general source geometries will contain the quantities which depend only on the vertical properties of the medium as $k_{j}$, [GN-LH], and $(\partial F / \partial k)$. In particular, if the source can be represented as

$$
\left\langle p_{\mathbf{s}}(r, \theta)\right\rangle=\cos (m \theta) \int_{0}^{\infty} p_{\mathrm{s}} J_{\mathrm{m}}(k r) d k
$$


the solution would be

$$
\left\langle w_{0}(r, \theta)\right\rangle=-i \cos (m \theta) \int_{0}^{\infty} \frac{p_{\mathrm{s}}}{k} \frac{1}{A_{22}} \frac{[G N-L H]}{F_{\sigma}} J_{\mathrm{m}}(k r) d k .
$$

For most general pressure distributions on the Earth's surface we can integrate the solution for a point pressure source over the desired source area. The point pressure source is defined here as $p_{\mathrm{s}}=k / 2 \pi$, in order that $\left\langle p_{\mathrm{s}}(r)\right\rangle$ given in equation (4) be a delta function. Using equation (9), the solution for the point source is written

$$
\left\langle w_{0}(r, \theta)\right\rangle=\frac{-i}{2 \pi} \int_{0}^{\infty} \frac{[G N-L H]}{F_{\mathrm{R}}} J_{0}(k r) d k .
$$

The solution for a distributed surface source, $\left\langle p_{\mathrm{s}}(r, \theta)\right\rangle$, is then given by the integral over the surface $S$ as

where we define

$$
\left\langle w_{0}(r, \theta)\right\rangle=\int_{S}\left\langle p_{\mathbf{s}}\left(r_{0}, \theta_{0}\right)\right\rangle G\left(\mathbf{r} \mid \mathbf{r}_{0}\right) d S_{0}
$$

$$
\begin{gathered}
G\left(\mathbf{r} \mid \mathbf{r}_{0}\right)=\frac{-i}{2 \pi} \int_{0}^{\infty} \frac{[G N-L H]}{F_{\mathrm{R}}} J_{0}(k R) d k \\
R=\left|\mathbf{r}-\mathbf{r}_{0}\right|
\end{gathered}
$$

and $\mathbf{r}$ is the position vector in the $(r, \theta)$ plane. An example of this technique is given in Appendix A for a disc source, which can be verified by equation (4).

\section{Surface overpressure models}

The distributed sea level overpressures are assumed to result from a spherical wave incident at a rigid surface from a point source at a height $D$ above the surface. The spherical wave front is assumed to expand with velocity $\alpha$. That is, the overpressure at a distance $r$ from ground zero is

$$
\left\langle p_{\mathrm{s}}(r)\right\rangle=2 p_{0 \mathrm{~s}} \frac{\exp \left(-i \omega R_{0} / \alpha\right)}{R_{0}}
$$

where

$$
R_{0}^{2}=D^{2}+r^{2} .
$$

The assumed pressure time history is the Glasstone pressure pulse (Glasstone 1962):

$$
\left[p_{\mathrm{e}}\left(a_{\mathrm{s}}, t\right)\right]= \begin{cases}p_{\mathrm{as}}\left(1-\tau / T_{+ \text {as }}\right) \exp \left[-\tau / T_{+\mathrm{as}}\right], & \tau>0 \\ 0, & \tau<0\end{cases}
$$

where $\tau=t-a_{\mathrm{s}} / \alpha . p_{\text {as }}$ is the peak overpressure and $T_{+ \text {as }}$ is the positive phase duration at $R_{0}=a_{s}$. Expressing (13) as an outgoing spherical wave and taking the Fourier time transform, we obtain

$$
\left\langle p_{\mathrm{e}}\left(R_{0}\right)\right\rangle=\frac{i \omega p_{\mathrm{as}} a_{\mathrm{s}}}{\left(\omega^{2}+b_{\mathrm{s}}{ }^{2}\right) R_{0}} \exp \left\{-i\left[\omega R_{0} / \alpha+2 \tan ^{-1}\left(\omega / b_{\mathrm{s}}\right)\right]\right\}
$$

where $b_{\mathrm{s}}=1 / T_{\text {+as }}$. From equations (13) and (14) we see that

$$
p_{0 \mathrm{~s}}=\frac{i \omega p_{\mathrm{as}} a_{\mathrm{s}}}{\omega^{2}+b_{\mathrm{s}}^{2}} \exp \left[-i 2 \tan ^{-1}\left(\omega / b_{\mathrm{s}}\right)\right]
$$


Using the Sommerfeld integral, we can express $\left\langle p_{s}(r)\right\rangle$ in the integral form of equation (4) as

$$
\left\langle p_{\mathrm{s}}(r)\right\rangle=-i 2 p_{0 \mathrm{~s}} \int_{0}^{\infty} \frac{\exp (-i v D)}{v} k J_{0}(k r) d k
$$

where

$$
v^{2}=\left(\frac{\omega}{\alpha}\right)^{2}-k^{2}
$$

and thus

$$
p_{\mathrm{s}}=\frac{2 \omega p_{\mathrm{as}} a_{\mathrm{s}} k}{v\left(\omega^{2}+b_{\mathrm{s}}{ }^{2}\right)} \exp \left\{-i\left[v D+2 \tan ^{-1}\left(\omega / b_{\mathrm{s}}\right)\right]\right\} .
$$

We obtain the ocean bottom Rayleigh wave displacement in the far field by substituting equation (15) into equation (10) and transforming back into the time domain:

$$
\begin{aligned}
{\left[w_{\mathrm{a}}\right]_{R_{j}}=} & \left(\frac{2}{\pi}\right)^{\frac{1}{2}} \int_{-\infty}^{\infty} \frac{\omega p_{\mathrm{as}} a_{\mathrm{s}} k_{j}{ }^{-\frac{1}{2}}}{A_{22} v\left(\omega^{2}+b_{\mathrm{s}}{ }^{2}\right)} \frac{\exp [-\hat{\gamma}(\omega) r]}{\left[a_{\mathrm{e}} \sin \theta_{\mathrm{e}}\right]^{\frac{1}{2}}} \mathscr{A}_{\sigma} \\
& \times \exp \left\{i\left[\omega t-k_{j} r+3 \pi / 4-\phi_{\mathrm{s}}\right]\right\} d \omega
\end{aligned}
$$

where

$$
\phi_{\mathrm{s}}=v D+2 \tan ^{-1}\left(\omega / b_{\mathrm{s}}\right)
$$

and

$$
\mathscr{A}_{\sigma}=-i \frac{[G N-L H]}{\left(\hat{\partial} F_{\sigma} / \partial k\right)}
$$

and where we have used the asymptotic expansion for large arguments of the Hankel function, as well as approximation for the Earth's curvature, as in equation (3). The expression for the continental model can be obtained by setting $A_{22}=1$ and $A_{21}=0$ in equation (16).

\section{Source overpressure scaling}

The scaling of the outgoing wave (equation (13)) for the mass and energy injection source models for different yields and burst altitudes is done using the formulas in Glasstone (1962). Using values obtained from Glasstone (1957) for a $1 \mathrm{kT}$ typical air-burst nuclear explosion, the scale distance $a_{\mathrm{s}}$, peak overpressure $p_{\text {as }}$, and positive phase duration $T_{+ \text {as }}$ are given by:

$$
\begin{array}{rlrl}
a_{\mathrm{s}} & =\xi a_{0} & p_{\text {as }} & =\left[\frac{P_{\mathrm{s}}(D)}{P_{0}}\right] p_{\mathrm{a} 0} \\
T_{+\mathrm{as}}=\xi\left[\frac{\alpha_{0}}{\alpha}\right] T_{+\mathrm{a} 0} & \xi & =\left\{W\left[\frac{P_{0}}{P_{\mathrm{s}}(D)}\right]\right\}^{\frac{\xi}{5}} .
\end{array}
$$

Here, $W$ is the kT equivalent yield, $P_{0}$ and $a_{0}$ are the ambient pressure and acoustic velocity at sea level, and $P_{\mathrm{s}}$ and $a_{\mathrm{s}}$ are at the burst altitude. Except for the low burst altitudes, this scaling at the source altitude is not appropriate for a surface applied source where we need the sea-level peak overpressure, unlike Harkrider \& Flinn (1970) where the scaling of the outgoing wave is done at the same altitude as the source. 
For nuclear explosions in the intermediate altitude range, $10 \leqslant D \leqslant 100 \mathrm{~km}$, we use the weak shock overpressure relations derived by Murphy (1972):

$$
\begin{gathered}
p_{\text {as }} a_{\mathrm{s}}=0.2 h\left[P_{\mathrm{s}}(D) P_{0}\right]^{\frac{1}{2}} \frac{x \mathrm{e}^{x}}{b(x)} \\
T_{\text {+as }}=-0.0857 \frac{h}{\alpha} \frac{x b(x)}{(1+x+n)} \\
b(x)=\left[1-2(1+x+n) \mathrm{e}^{x} E_{1}(x)\right]^{\frac{1}{2}}
\end{gathered}
$$

where $E_{1}(x)$ is the exponential integral, $h$ is the scale height of an exponential atmosphere, and $x$ and $n$ are matching parameters for a shock wave calculation in a spherically symmetric exponential atmosphere (Lutzky \& Lehto 1968). The matching parameters are given graphically and in tabular form as a function of $\sigma_{h}$ in Murphy (1972). The parameter $\sigma_{h}$ is a function of source height and yield:

$$
\sigma_{h}=h\left[\frac{P_{\mathrm{s}}(D)}{29.4 W}\right]^{\frac{1}{3}}
$$

where $h$ is in kilometres, $P_{s}(D)$ in millibars, and $W$ in kT.

Equations (17) are the extrapolation relations using the Reed-Otterman analytic weak-shock theory (Reed 1959). As in Murphy (1972), we will restrict our calculations to source heights and yields for which the ratio of overpressure to ambient pressure on the ground is smaller than 0.1 .

\section{Isothermal atmosphere model}

Since the result for the atmosphere/solid-Earth system (equation (3)) is similar to the distributed overpressure result (equation (16)), we will compare the simple model of an isothermal gravitating atmosphere with the sea-level distributed source.

For a single-layer atmosphere of thickness $H$,

where

$$
\begin{gathered}
\tilde{A}_{21}=i \rho_{0} \exp (-\lambda H) \frac{\delta}{\omega^{3}} \frac{\sin (\bar{v} H)}{\bar{v}} \\
\tilde{A}_{22}=\exp (-\lambda H)[\cos (\bar{v} H)+f \sin (\bar{v} H)]
\end{gathered}
$$

and

$$
f=\left(\lambda-g / c^{2}\right) / \bar{v}
$$

$$
\delta=g^{2} k^{2}-\omega^{4}
$$

$\rho_{0}$ is the sea level ambient density. The $\tilde{A}_{s}$ elements are identical to the above, with $D$ replacing $H$.

Thus in equation (3) for the single layer system,

$$
\begin{aligned}
& \frac{1}{\rho_{\mathrm{s}}(D)}\left[\frac{p_{p}(D)}{\dot{w}_{0}}\right]=\exp (2 \lambda D) \frac{\delta}{\bar{v} \omega^{3}} \\
& \times \frac{\{[\exp (i \Psi)-\exp (-i \Psi)]+i f[\exp (i \Psi)+\exp (-i \Psi)]\}}{\exp (i \bar{v} H)\{1+\exp (-i 2 \bar{v} H)-i f[1-\exp (-i 2 \bar{v} H)]\}}
\end{aligned}
$$

where we have used the relations $\rho_{\mathrm{s}}(D)=\rho_{0} \exp (-2 \lambda D)$ and $\Psi=\bar{v}(H-D)$.

Neglecting the exponential terms involving the height of the atmosphere, we have:

$$
\frac{1}{\rho_{\mathrm{s}}(D)}\left[\frac{p_{p}(D)}{\dot{w}_{0}}\right] \simeq \frac{P_{0}}{P_{\mathrm{s}}(D)} \frac{\delta}{\bar{v} \omega^{3}} \exp \left[-i \bar{v} D+i 2 \tan ^{-1} f\right] .
$$


For the range of frequencies and phase velocities associated with Rayleigh waves, the neglected exponential terms represent phase and group delays for waves which have travelled at least once to the top of the atmosphere. As $H$ becomes larger, their contribution to the Rayleigh wave arrives increasingly later than the contribution of the direct wave from source to ground, which is represented in this approximation.

Harkrider \& Flinn (1970) found that the atmosphere had little numerical effect on the medium response $\mathscr{A}_{\mathrm{e}}$; i.e. for the ocean system, $\mathscr{A}_{\mathrm{e}} \simeq \mathscr{A}_{\sigma}$ and for the continent $\mathscr{A}_{\mathrm{e}} \simeq \mathscr{A}_{\mathrm{R}}$. Thus, comparing equation (3) with the continent solution corresponding to equation (16), the integrands differ by a factor $S_{\mathrm{R}}$ for the surface and $S_{\mathrm{e}}$ for the energy injection source, where

$$
S_{\mathrm{R}}=p_{\mathrm{as}} a_{\mathrm{s}} \frac{\exp (-v D)}{v} \text { and } S_{\mathrm{e}}=p_{\mathrm{a} 0} a_{\mathrm{s}} \frac{\exp (-\bar{v} D)}{\bar{v}} \exp \left(i 2 \tan ^{-1} f\right)
$$

after approximating $\delta$ in the Rayleigh wave $(\omega, k)$ range by

$$
\delta=g^{2} k^{2}-\omega^{4} \simeq-\omega^{4} .
$$

For $g=0$ the atmosphere is no longer exponential and we have $f=0$ and $\bar{v}=v$. The two factors are now equal since $p_{\mathrm{as}}$ and $p_{\mathrm{a} 0}$ differ only in the ratio of ambient pressures at the source altitude and sea level. If we use the energy injection source as our standard because of its success in acoustic-gravity wave prediction, the correct applied overpressure, $p_{\text {as }}$, for equation (13) should be the sea level value, $p_{\mathrm{a} 0}$.

\section{Numerical results}

\section{Earth and atmosphere models}

Two Earth models were used throughout this study. The Gutenberg continental model was taken from Ben-Menahem \& Harkrider (1964, p. 2610) and the AndersonToksöz oceanic model was taken from Harkrider \& Anderson (1966, p. 2970). The velocities and densities of these models are shown in Fig. 1.

The atmosphere was represented by isothermal layers comprising the standard ARDC atmosphere (Wares et al. 1960) and is extensively described by Press \& Harkrider (1962) and Harkrider (1964). The temperature profile for the atmospheric model is shown in Fig. 2. The velocities $c$ and $U$, and the medium responses $\mathscr{A}_{\mathrm{e}}$ for the atmosphere-continental model and for the atmosphere-oceanic model, as calculated by the atmosphere-earth coupled system dispersion program $\mathrm{HASH}$, are tabulated in Table 1.

Misleading values of Rayleigh dispersion for the coupled system are obtained when the Rayleigh wave roots are apparently intersected by the roots of the atmospheric acoustic modes; these occur along lines of nearly infinite slope in this region of the velocity-period plane. Fig. 3 shows the structure of the phase velocity curves at one such point of intersection, and Fig. 4 shows the group velocities calculated for the roots shown in Fig. 3 . The normalized atmospheric eigenfunction $\dot{w}_{m} / \dot{w}_{0}$ is additional evidence for this phenomenon; Fig. 5 shows $\dot{w}_{m} / \dot{w}_{0}$ at period $41.875 \mathrm{~s}$ near a point of intersection, and $T_{+1}$ and $T_{-1}$ just $0.3125 \mathrm{~s}$ above and below $T_{0}$. The periods chosen for the Rayleigh wave synthesis described here were required to have appropriate kinetic energy densities in the bottom layer of the atmosphere. This criterion resulted in the best velocity and medium response values for our models. The group velocities for these chosen periods are listed in Table 1.

\section{Anelastic and scattering attenuation coefficient}

The amplitude decay coefficients $\hat{\gamma}_{\mathrm{A}}(\omega)$ used here were taken from a study by Alewine (1972). These values differ appreciably from those used in Harkrider \& 


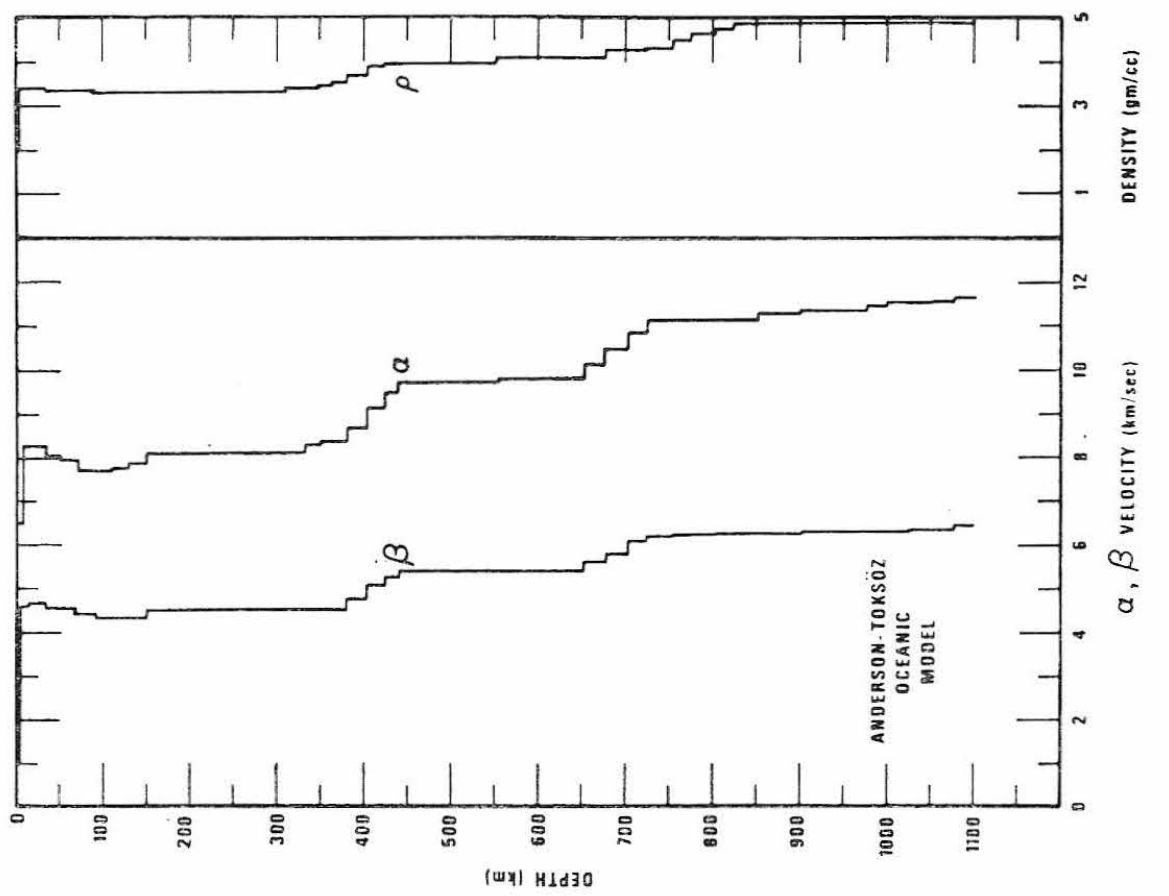

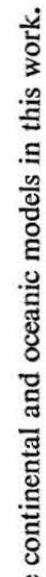

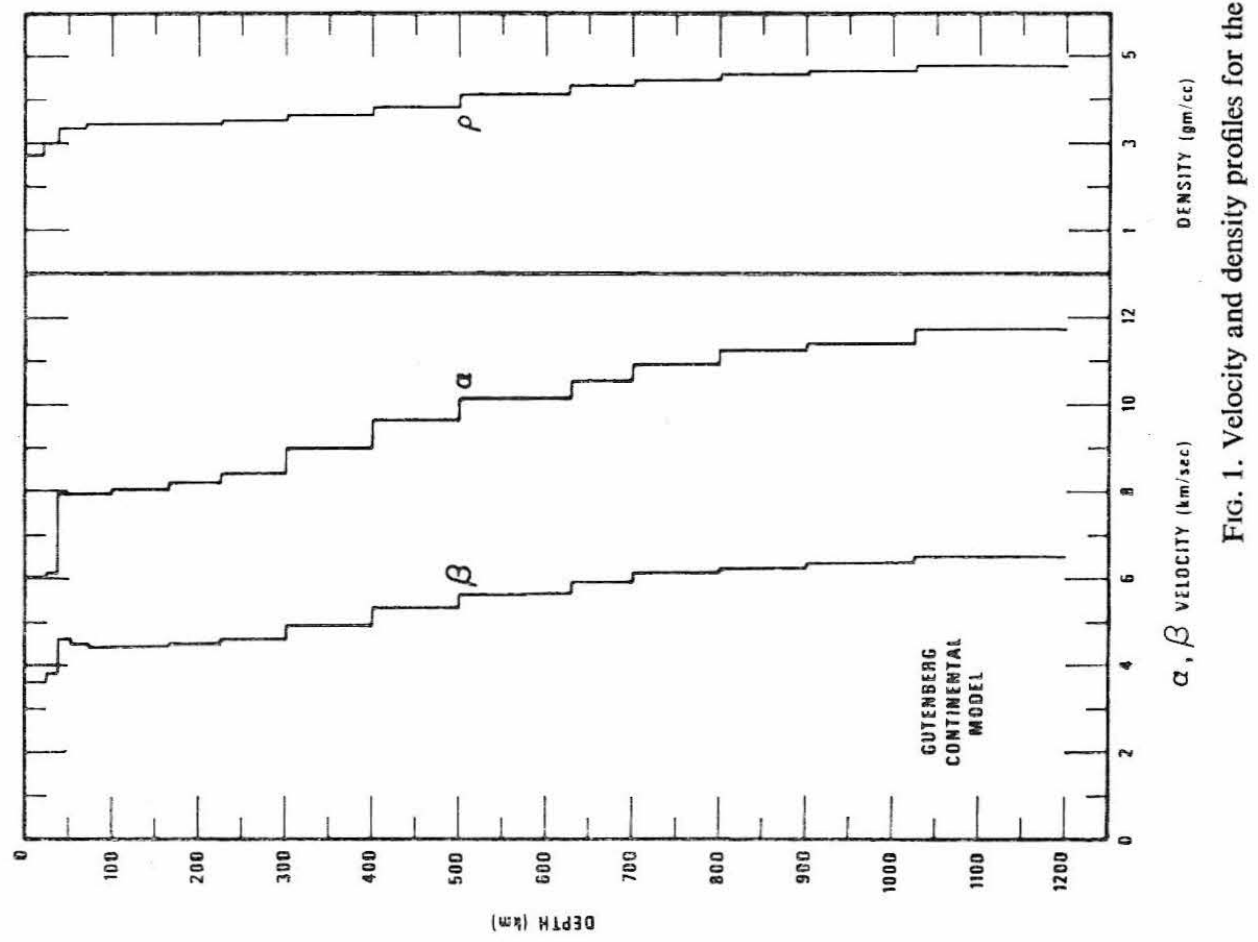




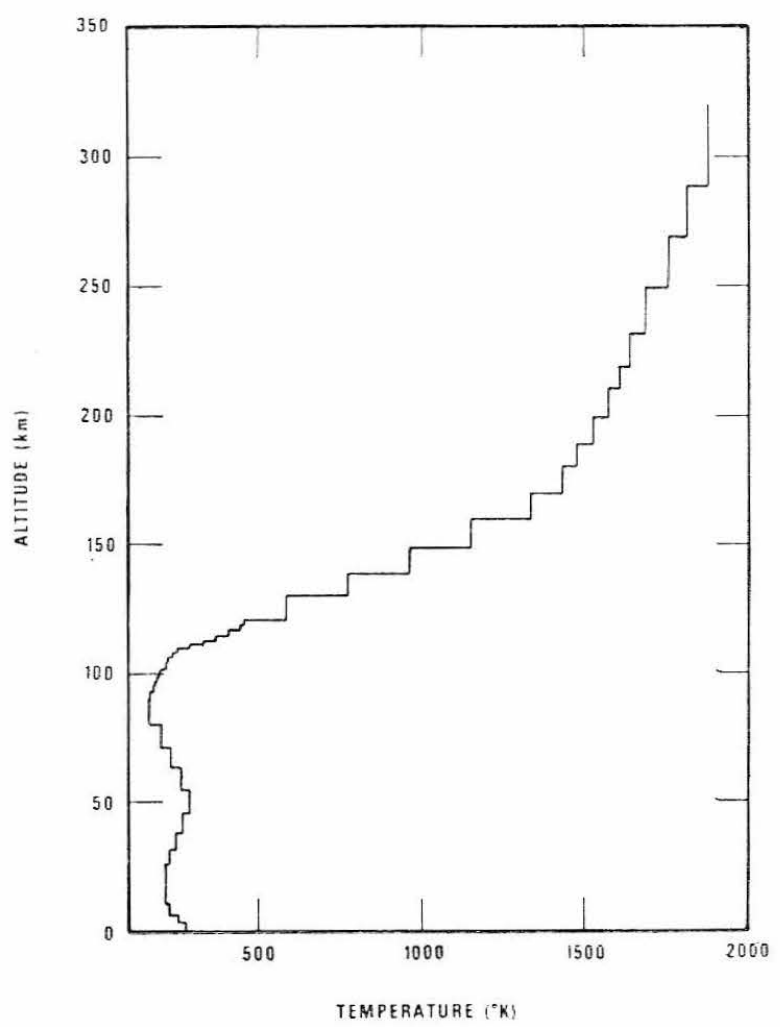

FIG. 2. Temperature profile in the atmosphere.

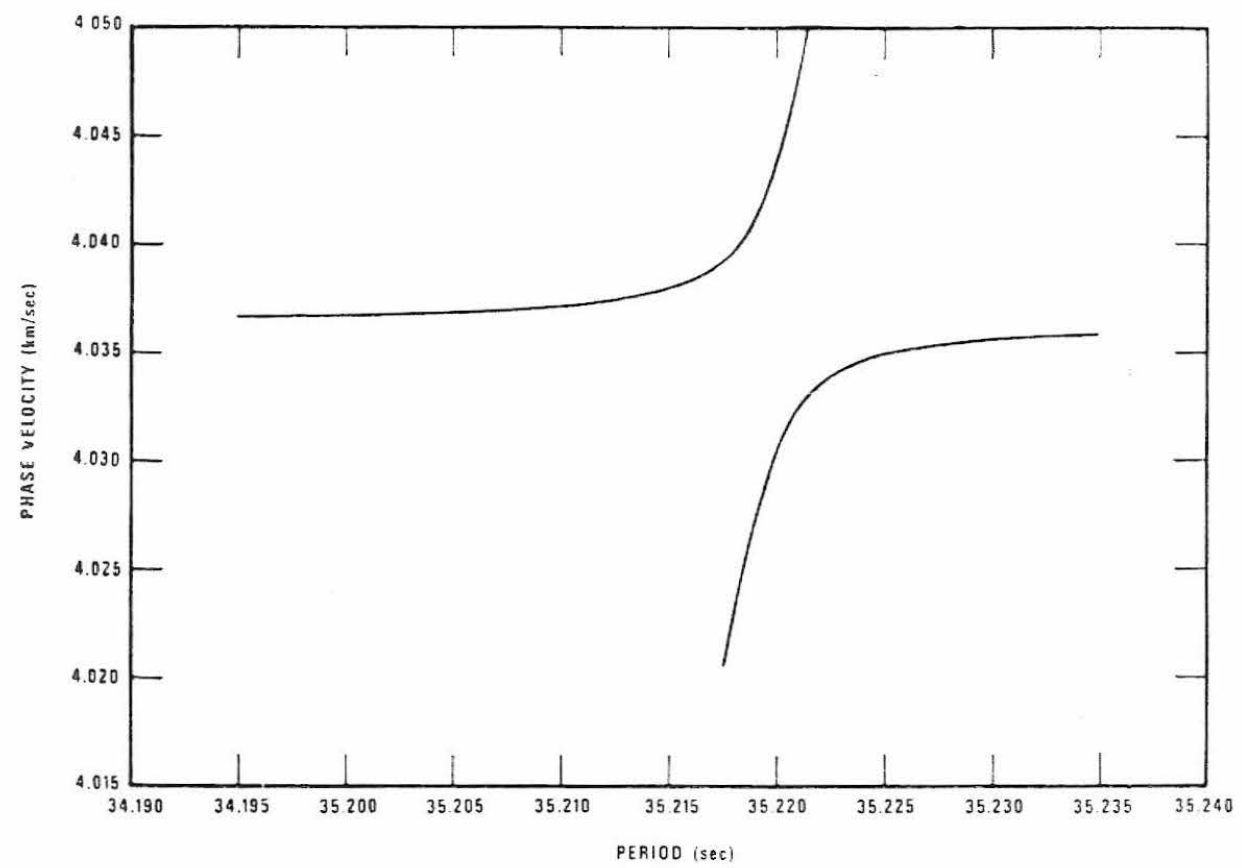

FIG. 3. Region of the $c-T$ plane where the fundamental mode Rayleigh roots and the higher-mode acoustic roots intersect. 


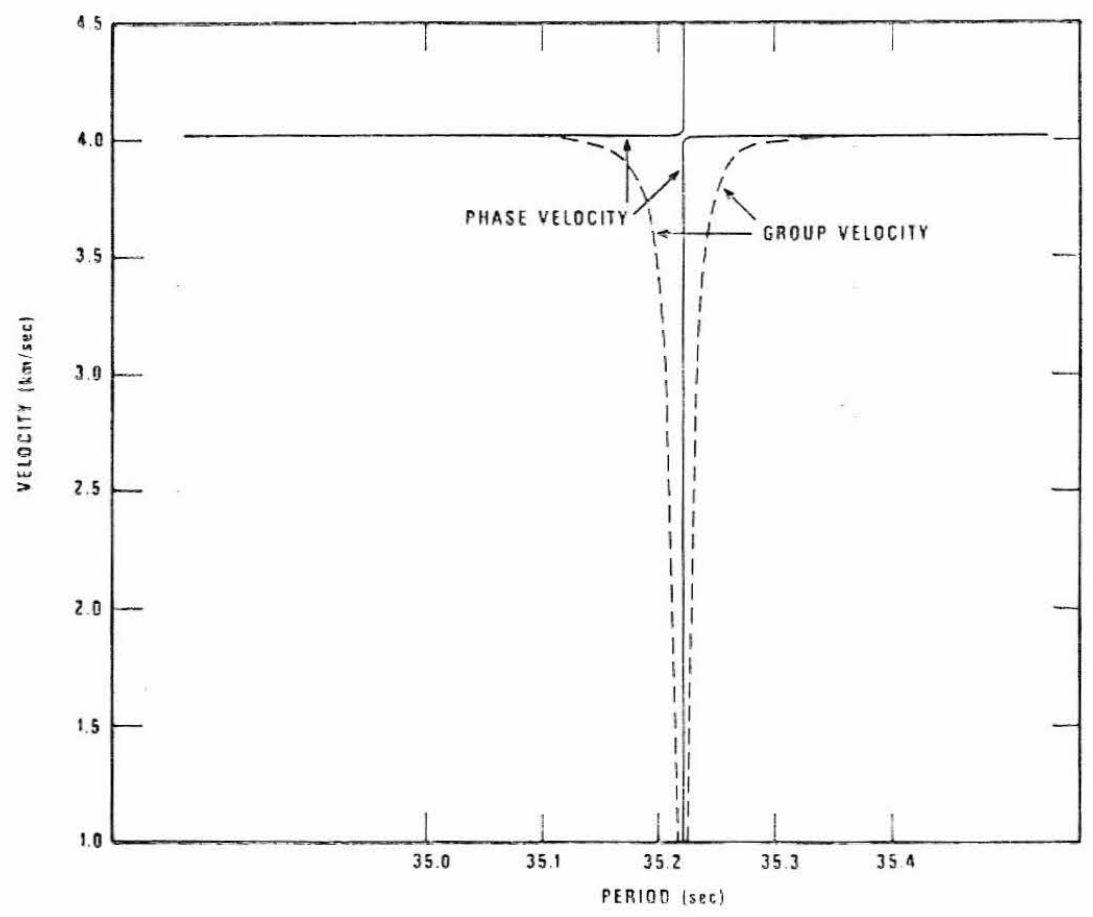

FIG. 4. Phase and group velocities for the region shown in Fig. 3.

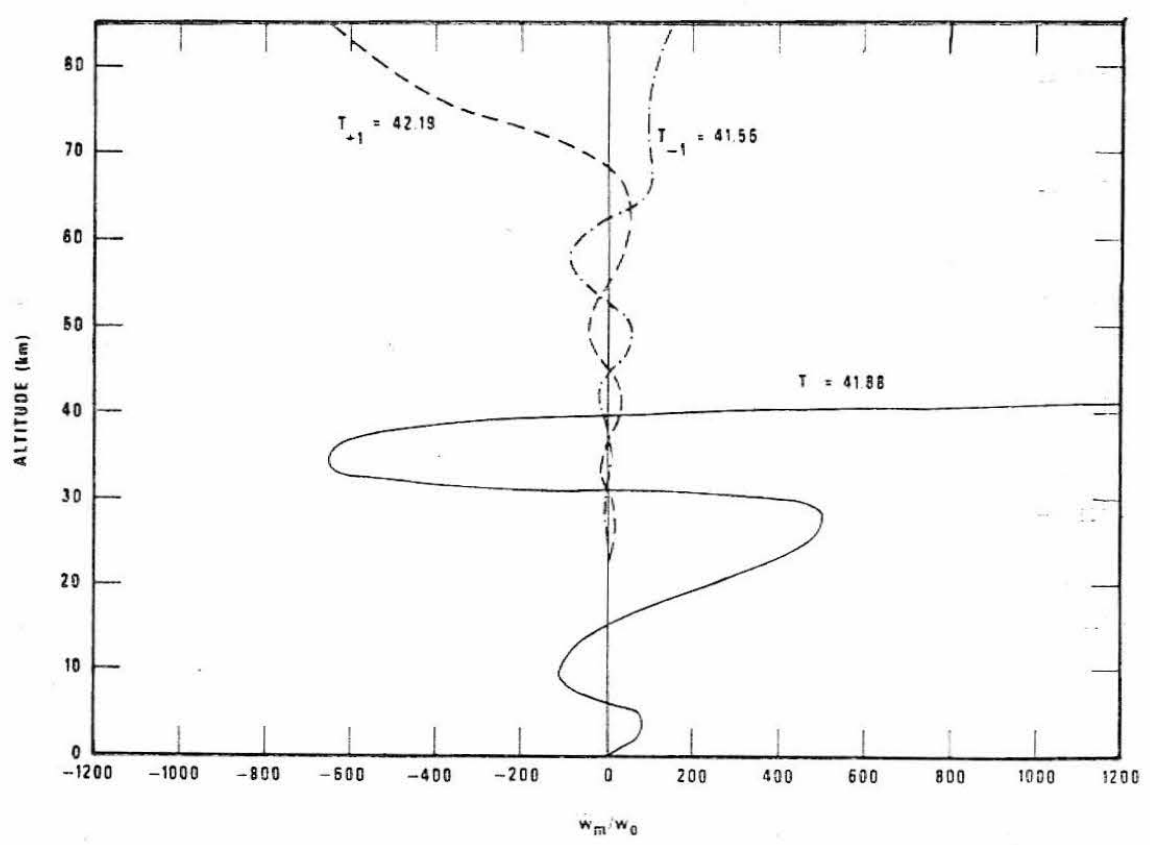

FIG. 5. Normalized vertical particle velocity in the atmosphere. 

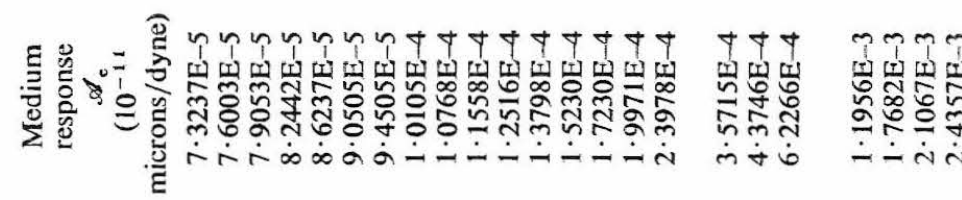

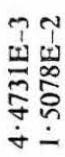

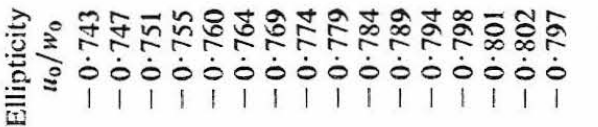

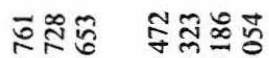

\&

i 11 i 1 i 


\section{Table 2}

\section{Peak amplitude corrections for different attenuation factors}

$\begin{array}{lc}\begin{array}{c}\text { Attenuation } \\ \text { coefficient }\end{array} & T_{\mathrm{m}}(\mathrm{s}) \\ \hat{\gamma}_{0}=0 & 21 \\ \hat{\gamma}_{\mathrm{A}} & 46 \\ \hat{\gamma}_{\mathrm{B}-\mathrm{M}} & 21 \\ & 46 \\ & 21 \\ & 46\end{array}$

$\begin{array}{cc}\begin{array}{c}\text { Synthesized } \\ \text { waveform peak } \\ \text { amplitude }\end{array} & \begin{array}{c}\text { Amplitudes } \\ \text { calculated from }\end{array} \\ \begin{array}{c}\hat{\gamma}_{\text {A }} \text { waveform } \\ 173\end{array} & 123 \\ 74 & 75 \\ 64 & - \\ 39 & - \\ 4 & 5 \\ 17 & 18\end{array}$

Flinn (1970), $\hat{\gamma}_{\mathrm{B}-\mathrm{M}}(\omega)$, which were obtained from a linear extrapolation of the longperiod observations of Ben-Menahem (1965).

The $\hat{\gamma}_{\mathrm{A}}(\omega)$ values are based on observations by Tryggvason and by Gutenberg and Richter (Alewine 1972) for continental paths. We have used the $\hat{\gamma}_{\mathrm{A}}(\omega)$ in the Rayleigh wave calculations for both continental and oceanic models. If other coefficients $\hat{\gamma}_{0}(\omega)$ are preferred, then the peak Rayleigh wave amplitudes reported here could be corrected by

$$
\exp \left[\hat{\gamma}_{\mathrm{A}}\left(\omega_{m}\right)-\hat{\gamma}_{0}\left(\omega_{m}\right)\right] r \quad \text { where } \omega_{m}=\frac{2 \pi}{T_{m}}
$$

and $T_{m}$ is the period of the peak amplitude. If the spectral decay factor with the coefficients $\hat{\gamma}_{0}(\omega)$ causes the peak amplitude to occur at a different time within the Rayleigh wave train, corresponding to a different group arrival, then the best correction procedure (the only procedure if no wave form is given) is to redo the synthesis calculation.

As an illustration of these corrections we have calculated waveforms for a $100 \mathrm{kT}$ energy injection source at an altitude of $18.6 \mathrm{~km}$ and epicentral distance of $10000 \mathrm{~km}$

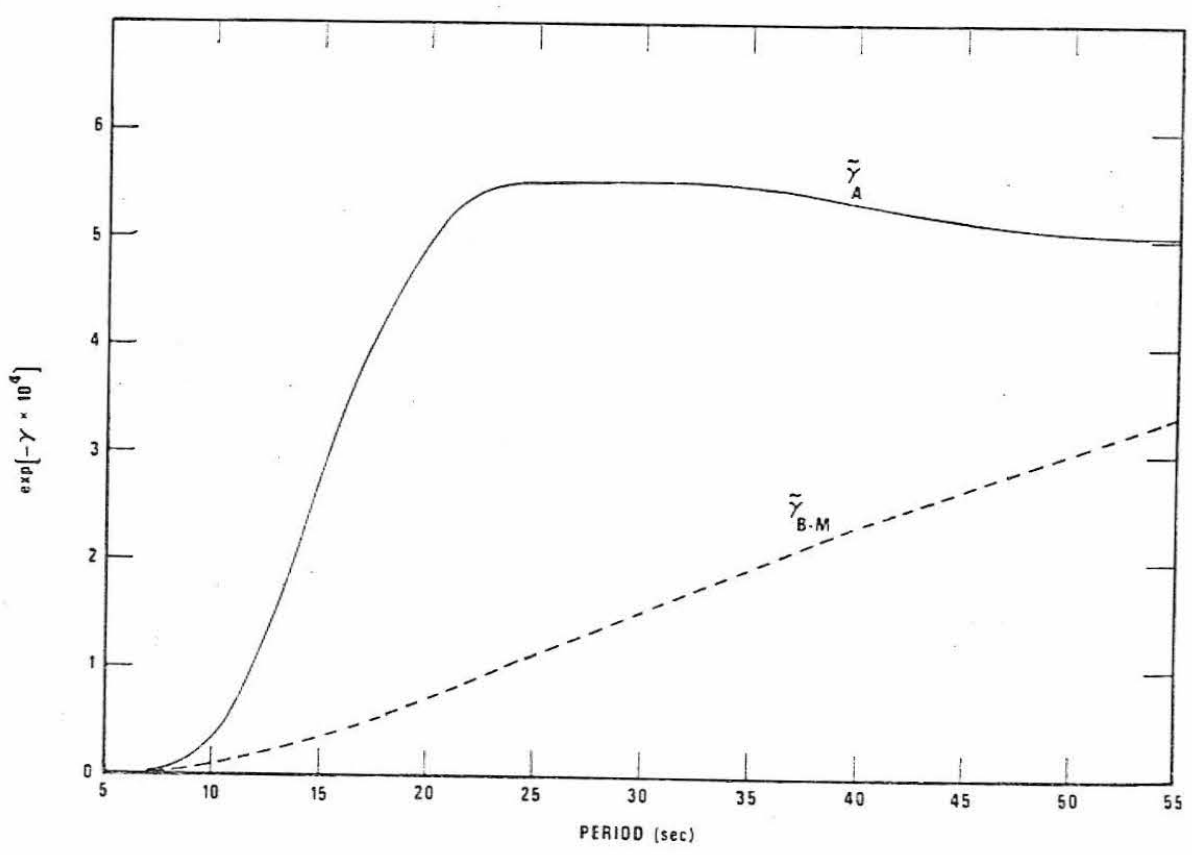

FIG. 6. Spectral decay factors $\exp \left(-\hat{\gamma}_{\mathrm{A}} \Delta\right)$ and $\exp \left(-\hat{\gamma}_{\mathrm{B}-\mathrm{M}} \Delta\right)$ for $\Delta=10000 \mathrm{~km}$ epicentral distance. 

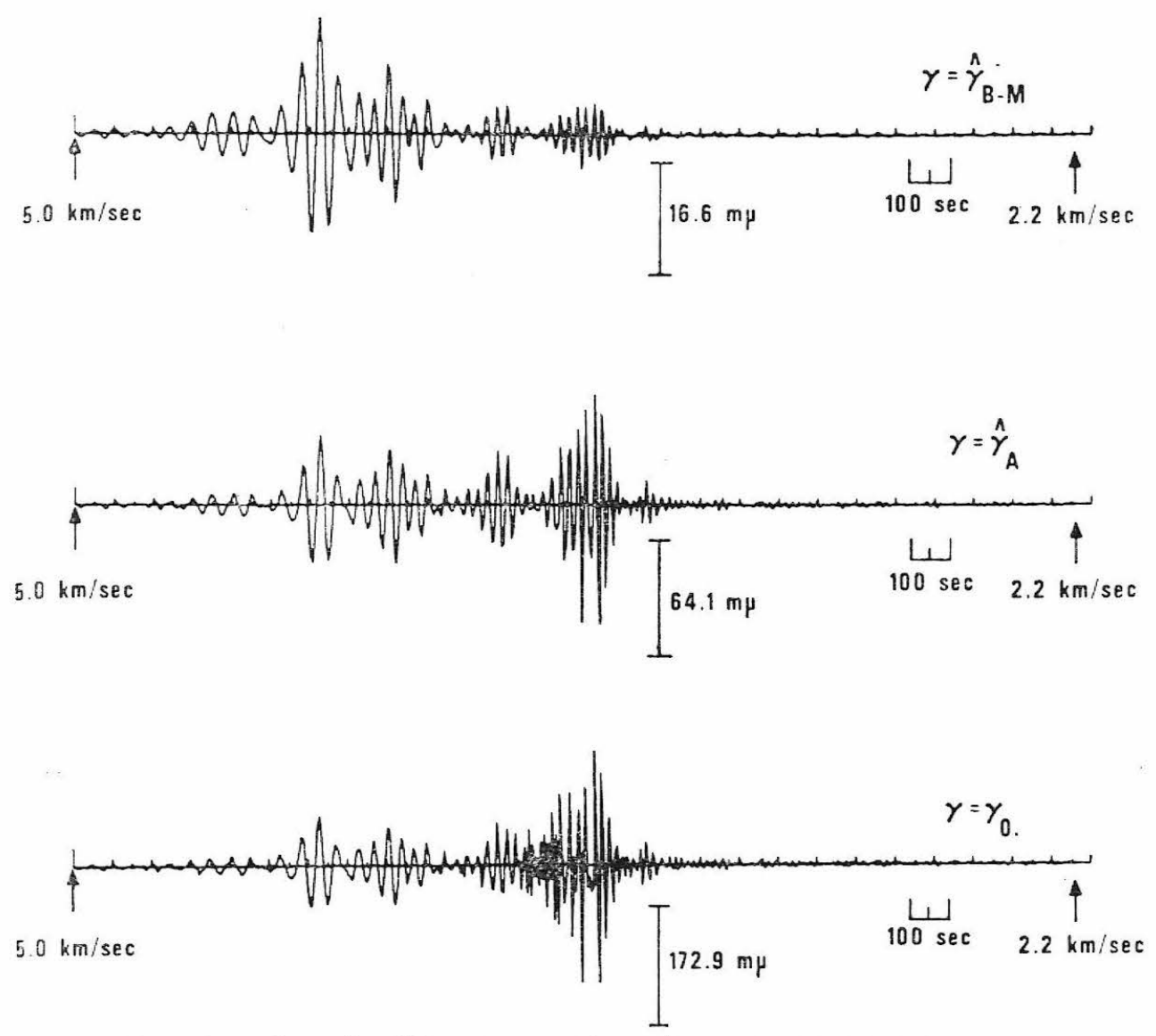

FIG. 7. Waveforms for different spectral decay factors described in text. Source altitude is $18.6 \mathrm{~km}$, yield is $100 \mathrm{kT}$, and epicentral distance is $10000 \mathrm{~km}$.

for $\hat{\gamma}_{\mathrm{B}-\mathrm{M}}(\omega), \hat{\gamma}_{\mathrm{A}}(\omega)$, and $\hat{\gamma}_{0}(\omega)=0$. The spectral decay factors for a $10000 \mathrm{~km}$ path are shown in Fig. 6. We see in Fig. 7 that the peak amplitudes for $\hat{\gamma}_{\mathrm{A}}$ and $\hat{\gamma}$ occur at $T_{m}=21 \mathrm{~s}$, but for $\hat{\gamma}_{\mathrm{B}-\mathrm{M}}$ at $T_{m}=46 \mathrm{~s}$. Table 2 shows a comparison between the measured amplitudes and the amplitudes calculated by making corrections to the measured $\hat{\gamma}_{\mathrm{A}}$ waveform.

The poor comparison at $21 \mathrm{~s}$ for $\hat{\gamma}_{0}=0$ (123 vs 173) might be the result of an anomalous Airy phase amplitude for this synthesis, since these comparisons were similar to those made for other source models. The peak amplitudes could also be calculated for different epicentral distances $r^{\prime}$ by multiplying the amplitude at distance $r$ by

$$
\exp \left[\hat{\gamma}\left(\omega_{m}\right)\left(r-r^{\prime}\right)\right]\left[\frac{r \sin \left(r / a_{\mathrm{e}}\right)}{r^{\prime} \sin \left(r^{\prime} / a_{\mathrm{e}}\right)}\right]^{\frac{1}{2}} .
$$

This correction is approximate and depends on the amount of dispersion of the wave train.

\section{Results for low source altitudes}

The theoretical seismograms presented by Harkrider \& Flinn (1970) were recalculated to correct for a missing factor of $(2 \pi)^{-1}$ and an incorrect altitude scaling for 


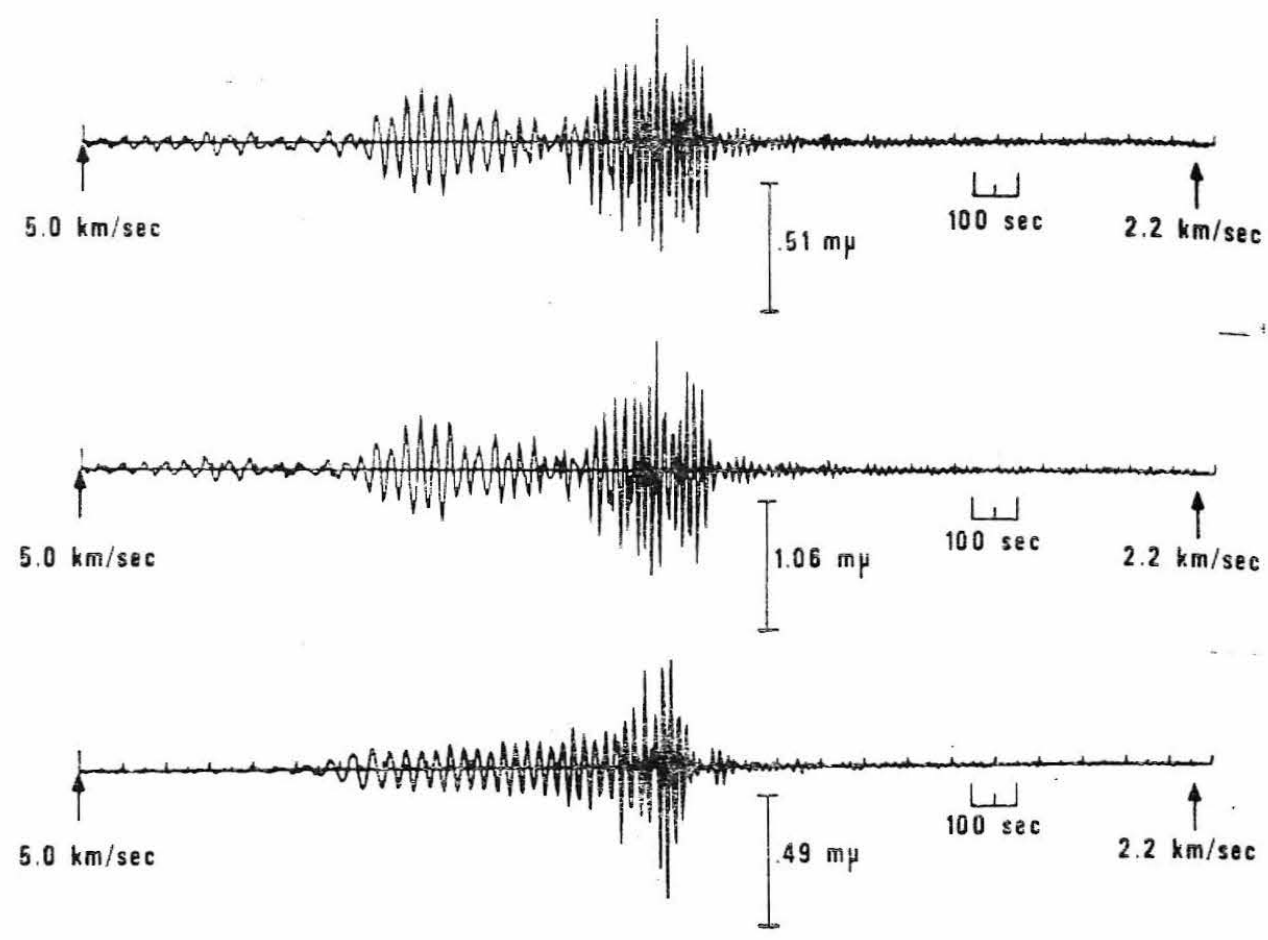

FIG. 8(a)

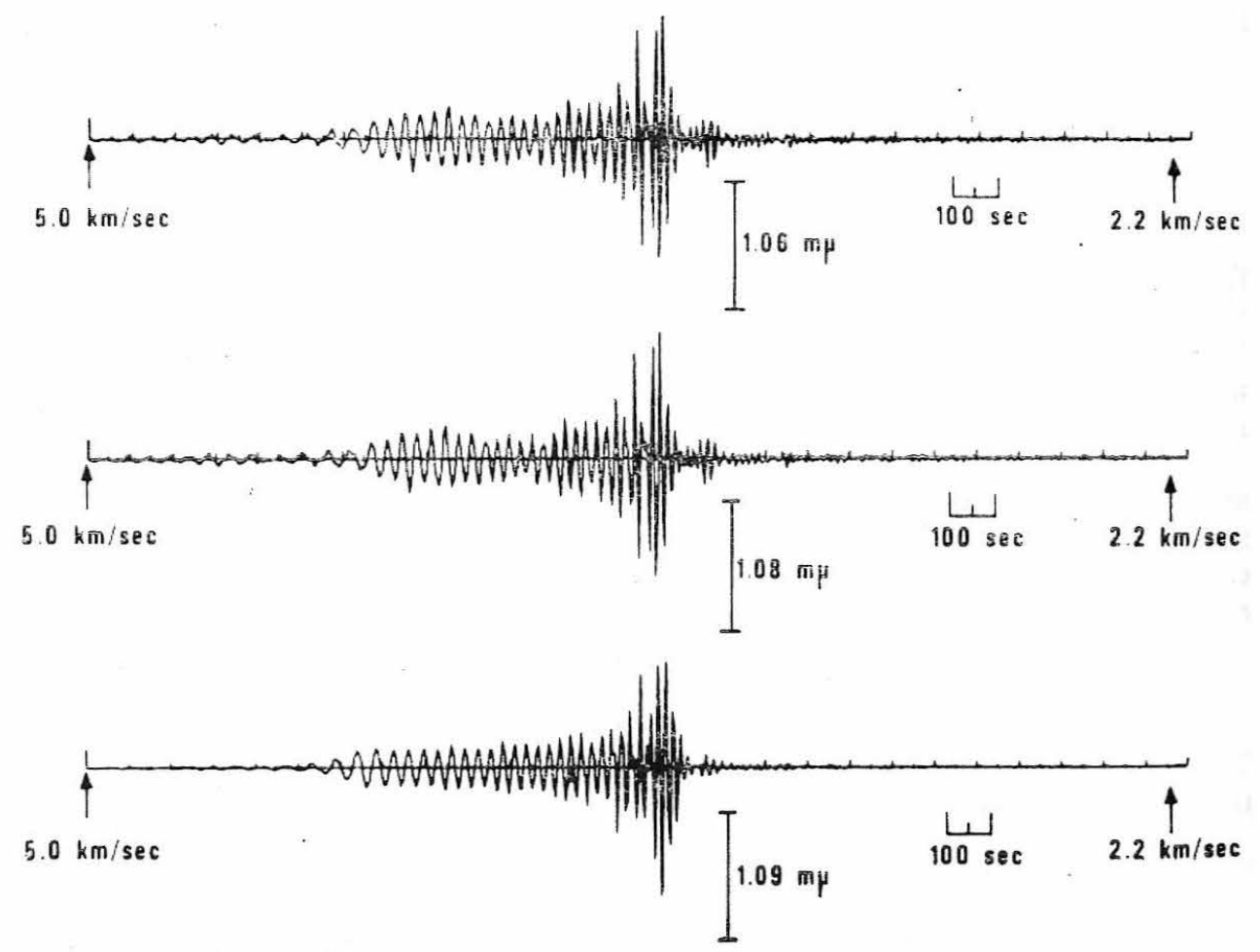

FIG. 8(b) 


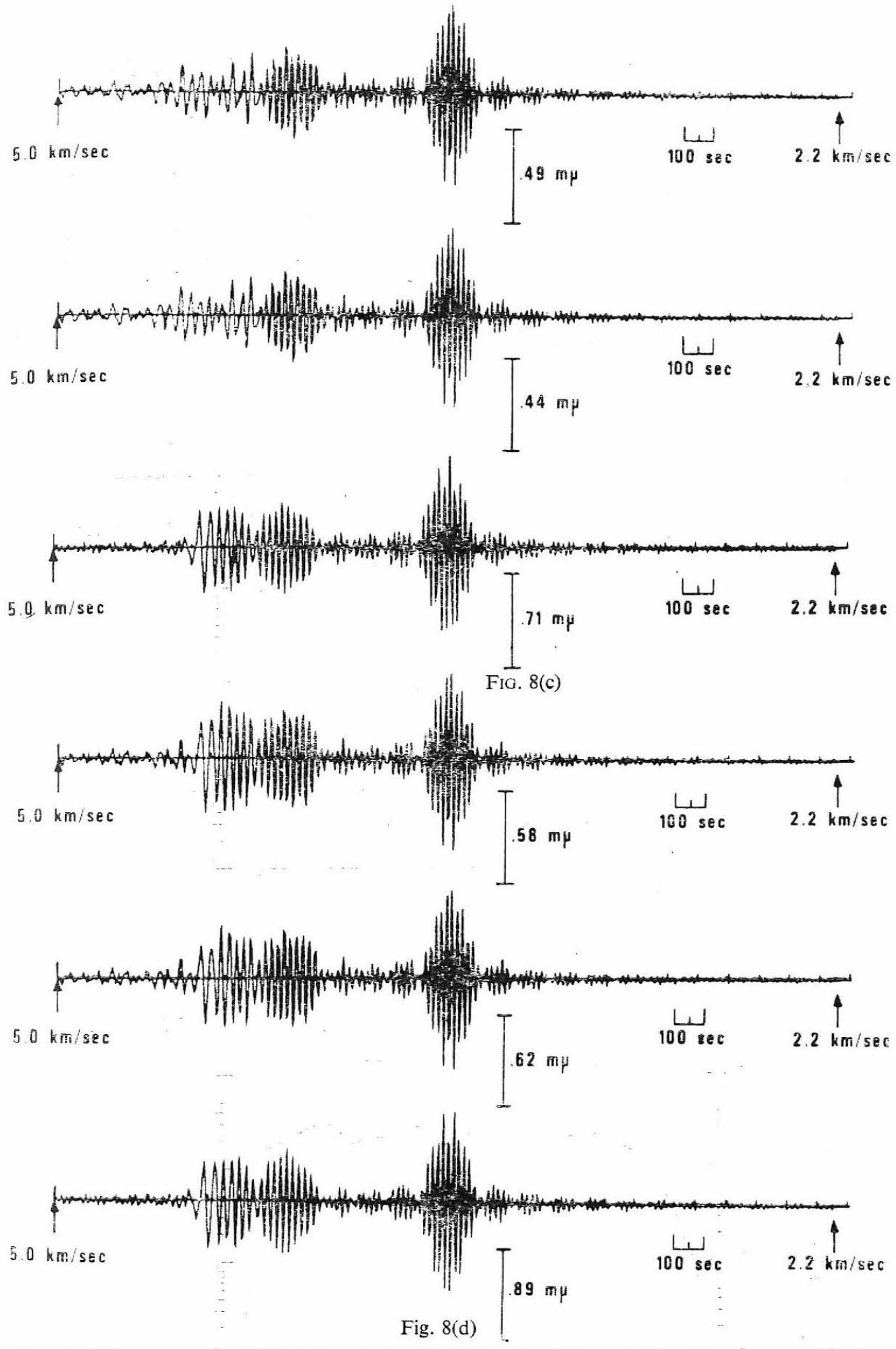

FIG. 8. Seismograms for $1 \mathrm{kT}$ yield and $\Delta=10000 \mathrm{~km}$ for different source types, source altitudes, and earth models: (a) continental model, source at $0.305 \mathrm{~km}$; (b) continental model, source at $1.83 \mathrm{~km}$; (c) oceanic model, source at $0.305 \mathrm{~km}$; (d) oceanic model, source at $1.83 \mathrm{~km}$. In each case the upper seismogram is for a mass-injection source, the middle seismogram for an energyinjection source, and the lower seismogram for a surface overpressure source. 
Table 3

Peak Rayleigh wave amplitudes $(m \mu)$ for $1 k T$ yield at low altitudes

Continental structure

Surface

Source height Mass

$(\mathrm{km})$

$0 \cdot 31$

$1 \cdot 83$

$3 \cdot 66$

$4 \cdot 88$ source

0.51

$1 \cdot 06$

$1 \cdot 04$

$1 \cdot 03$
Energy

source

0.49

1.08

0.97

$1 \cdot 08$
Oceanic structure

$\begin{array}{cc}\begin{array}{c}\text { Energy } \\ \text { source }\end{array} & \begin{array}{c}\text { Surface } \\ \text { overpressure } \\ \text { source }\end{array} \\ 0.44 & 0.71 \\ 0.62 & 0.69 \\ 0.86 & 0.71 \\ 0.52 & 0.72\end{array}$

the ocean model. Examples of seismograms are shown in Fig. 8 along with those calculated for the energy injection source (equation (3)) and the analytical surface overpressure Glasstone source (equation (16)). For these low source altitudes, as well

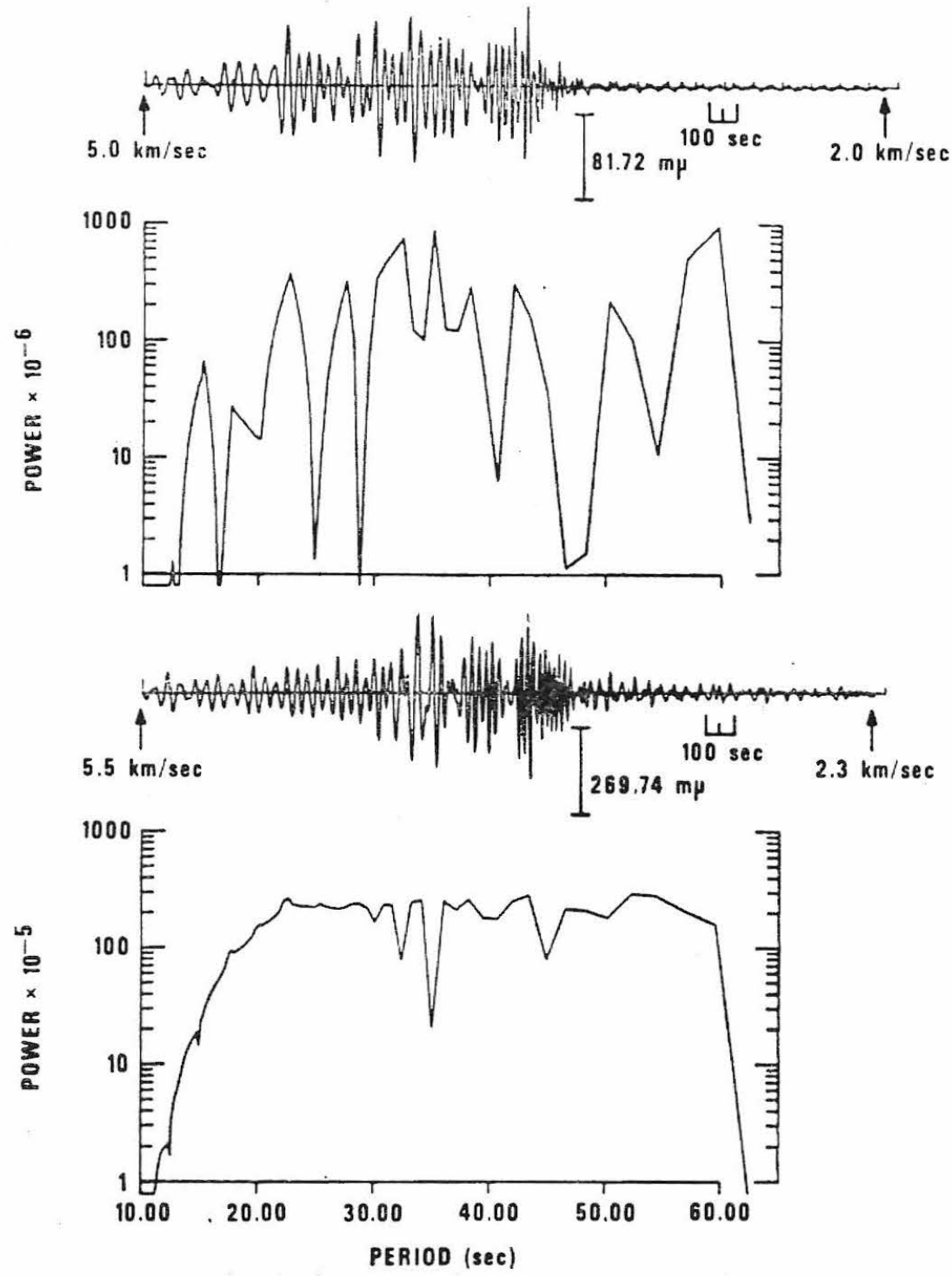

FIG. 9(a) 


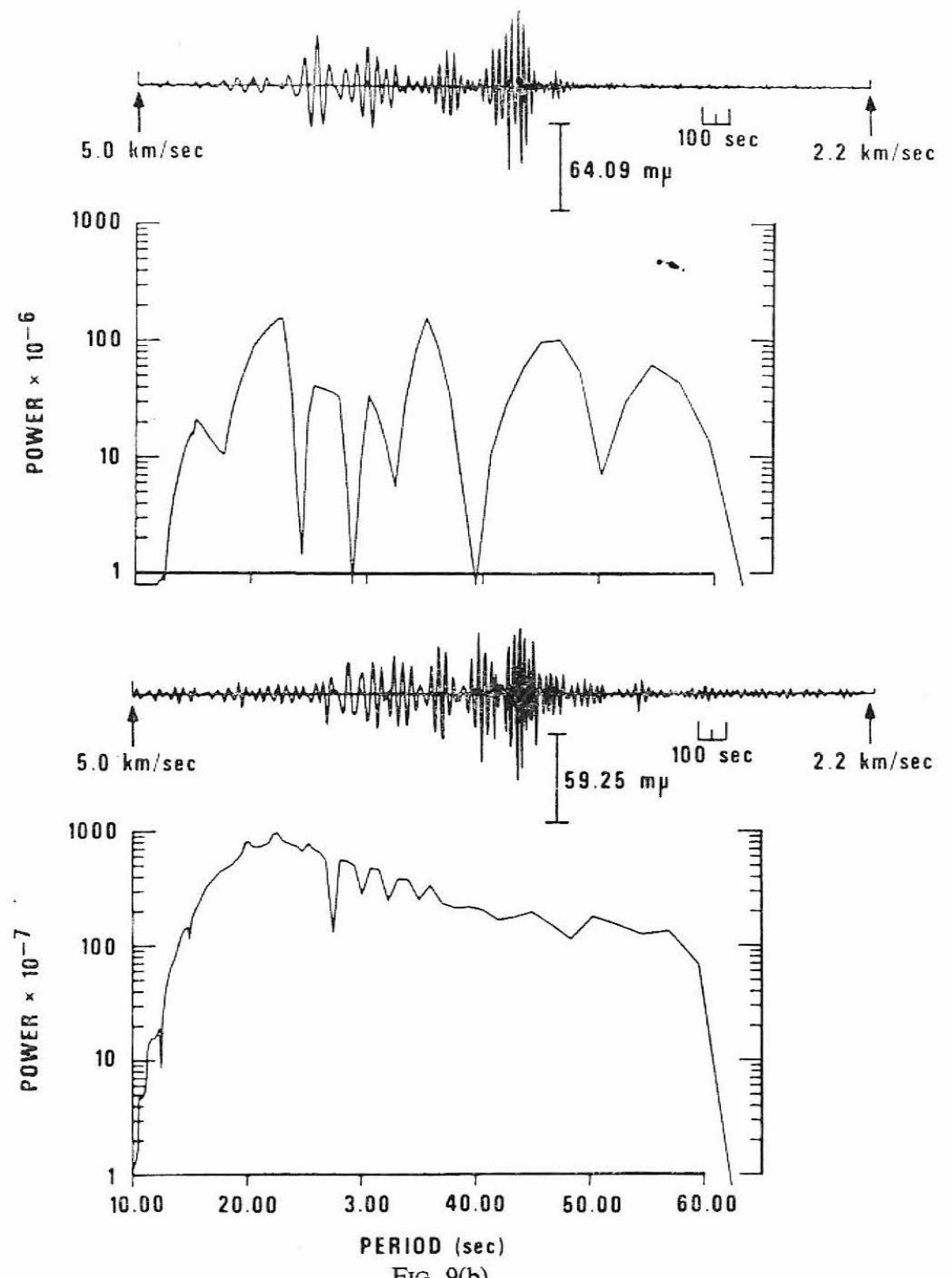



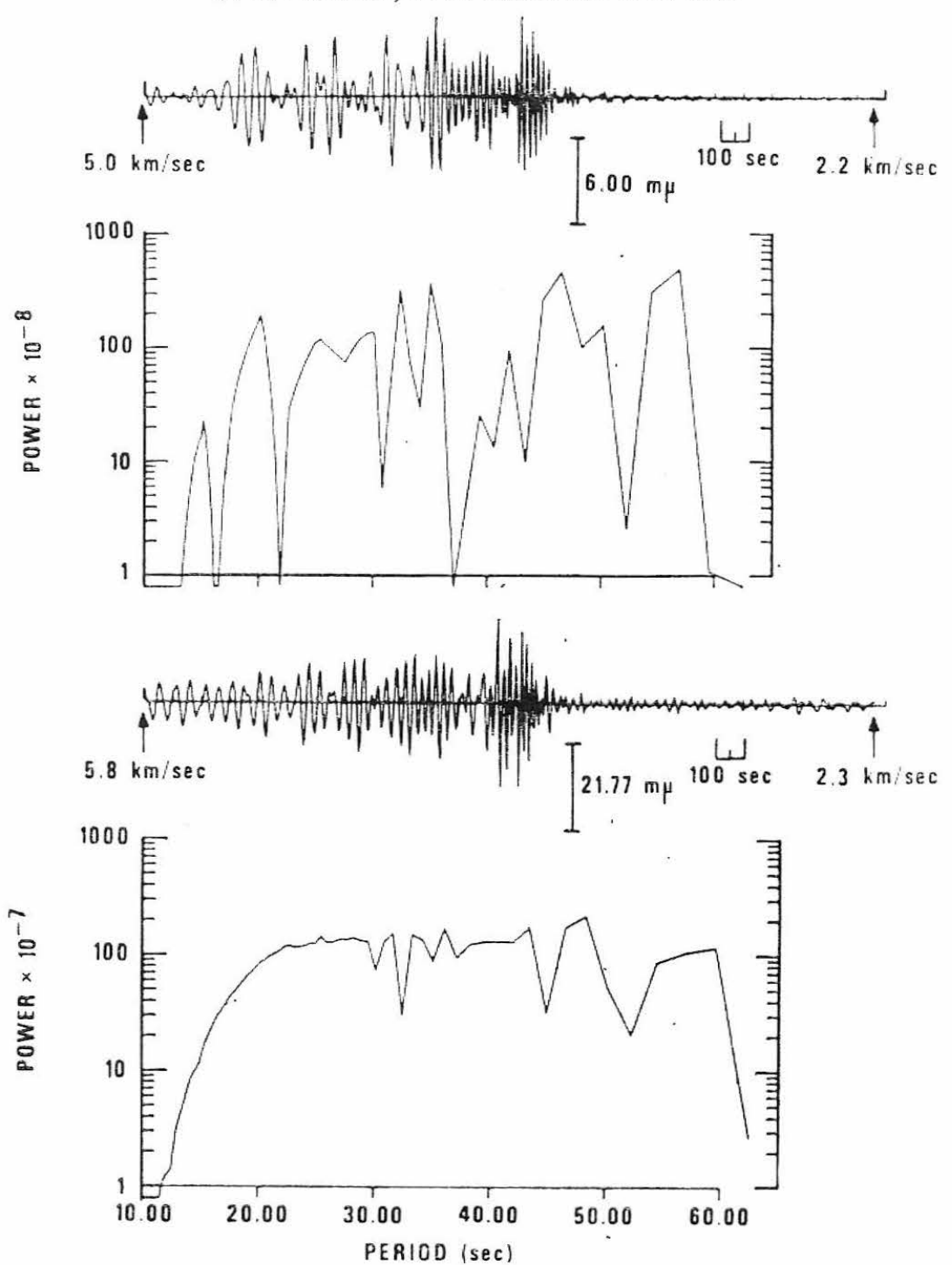

Fig. 9(c)

FIG. 9. Seismograms and amplitude spectra for intermediate source altitudes. Continental Earth model, for epicentral distance $\Delta=10000 \mathrm{~km}$. The upper figures show results for an energy injection source and the lower figures for a surface overpressure source: (a) $10 \mathrm{kT}$ at $82.3 \mathrm{~km}$ altitude; (b) $100 \mathrm{kT}$ at $18.6 \mathrm{~km}$ altitude; (c) $1 \mathrm{MT}$ at $51 \cdot 2 \mathrm{~km}$ altitude.

as for higher ones, the seismograms for the point mass and energy sources are practically indistinguishable. We have chosen the energy source as the standard because it gives better results for the acoustic-gravity waves. Table 3 shows a comparison of the peak amplitudes for the various low altitude source models.

\section{Intermediate source altitude results}

For source altitudes in the range $10-1000 \mathrm{~km}$ and yields of 10,100 and $1000 \mathrm{kT}$, we compared the Glasstone-scaled point energy source (equation (3)) and the Murphy (1972)-scaled surface overpressure source (equation (16)).

Typical waveforms generated by these source models are shown in Fig. 9. The 
smooth spectra for the surface source and the irregular-looking spectra for the source at altitude are predicted by the source terms in the integrands of the respective equations, and demonstrate the effect of multipathing in the atmosphere. The peak amplitudes for these source models, shown in Fig. 10, are nearly the same for the lowest source altitudes, but at higher altitudes the modified Sachs scaling calculated by Murphy (1972) predicts a larger Rayleigh wave amplitude.

\section{Uniform half-space models}

In 1971, Nickel and Whitaker presented a numerical technique in which the atmospheric explosion was modelled by a distributed source on the free surface of a solid half space. The elastic constants of the homogenecus half space were made to vary with the frequency in order to reproduce measured dispersion data. For a homogeneous half space the medium response is given by Harkrider (1970) as

$$
\begin{gathered}
\mathscr{A}_{\mathrm{R}}=\frac{r_{\alpha}{ }^{*}}{4 \rho V_{\mathrm{R}}{ }^{3}\left\{(\gamma-1)+\frac{\beta^{2}}{V_{\mathrm{R}}{ }^{2}} \frac{\gamma^{2}}{\alpha^{2}} \frac{\left[2 V_{\mathrm{R}}{ }^{2}-\alpha^{2}-\beta^{2}\right]}{(\gamma-1)^{2}}\right\}} \omega \\
\text { where } r_{\alpha}{ }^{*}=-\left(1-\frac{V_{\mathrm{R}}{ }^{2}}{\alpha^{2}}\right)^{\frac{1}{2}} \text { and } \gamma=2 \frac{\beta^{2}}{V_{\mathrm{R}}{ }^{2}} .
\end{gathered}
$$

Assuming a Poisson solid as in Nickel and Whitaker, with the Lamé constants equal, then the observed phase velocity of Rayleigh waves $V_{\mathrm{R}}(\omega)$ determines a frequency dependent shear, $\beta(\omega)$, and compressional velocity, $\alpha(\omega)$, given by

$$
V_{\mathbf{R}}=(2-2 / \sqrt{ } 3) \beta \text { and } \alpha=\beta \sqrt{ } 3 \text {. }
$$
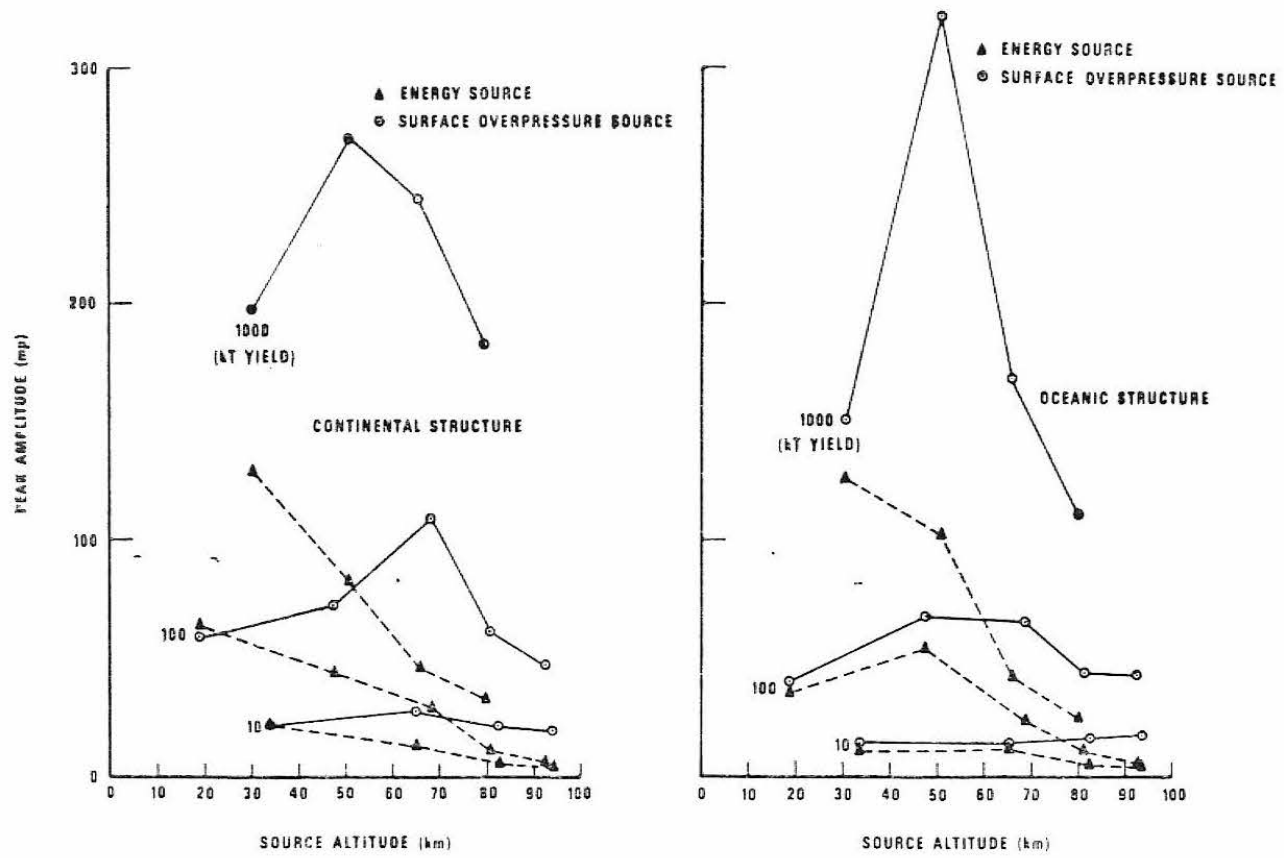

FIG. 10. Peak Rayleigh wave amplitude for intermediate source altitudes, for energy injection and surface overpressure sources. Left figure shows amplitudes for continental structure, right figure for oceanic structure. 
Thus the media response for this fictitious half space can be determined to within a factor of $\rho$, the density, which is assumed to be independent of frequency or

$$
\rho \mathscr{A}_{\mathrm{R}} \cong \frac{0.9745}{V_{\mathrm{R}}(\omega)^{3}} \frac{1}{T} \times 10^{-11} \text { microns } \mathrm{s}^{2} / \mathrm{cm}^{-4}
$$

where $V_{\mathrm{R}}$ is in kilometres per second and $T$ is in seconds. In Fig. 11, we show $\mathscr{A}_{\mathrm{R}}$ for various Earth structures including an oceanic model. If we assume a crustal density $\rho$ between 2.50 and 3.0 there is good agreement between the vertically inhomogeneous media responses and the approximate half space models of Nickel and Whitaker using our Rayleigh dispersion for a continent and ocean. This means that the technique of Nickel and Whitaker is valid for explosions over oceans as well as continents, a point questioned in Harkrider \& Flinn (1970).

\section{Yield and burst height diagnostics}

Analysis of theoretical seismograms to obtain a set of measurements capable of diagnosing yield and height of burst was attempted using the energy injection source model for both oceanic and continental layered structures. The peak amplitude, period of oscillation at the peak amplitude, and the surface wave magnitude were calculated (Table 4) at an epicentral distance of $10000 \mathrm{~km}$, yields of $1 \mathrm{kT}-10 \mathrm{MT}$,

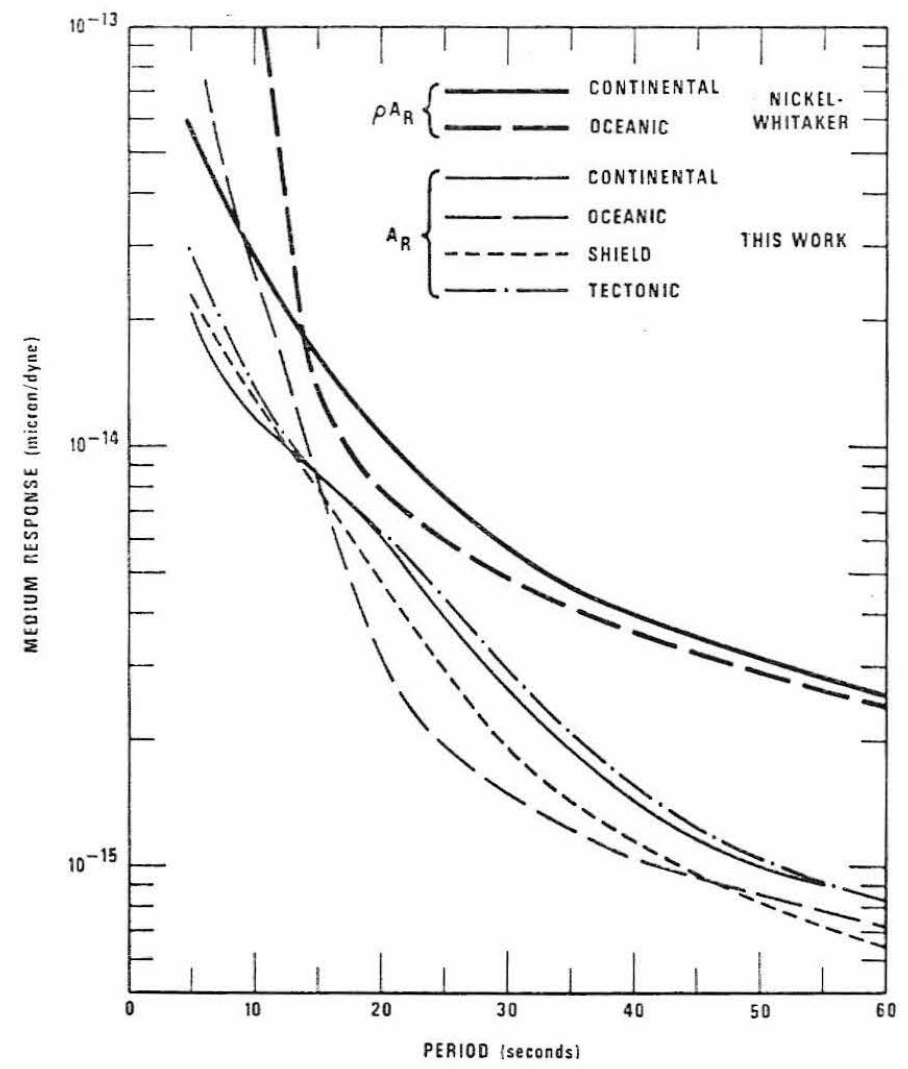

FIG. 11. Medium response as a function of period for four earth structures. Theoretical prediction of Nickel \& Whitaker shown for comparison and labelled $\rho \mathscr{A}_{\mathbf{R}}$. 


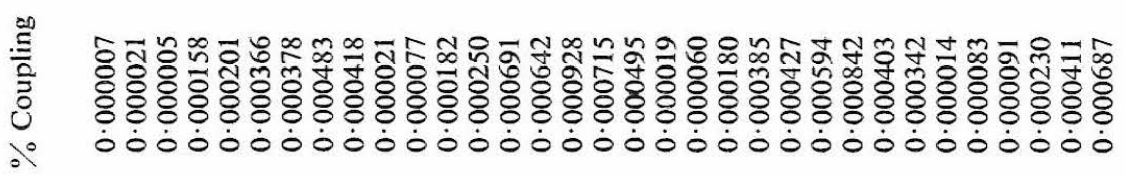

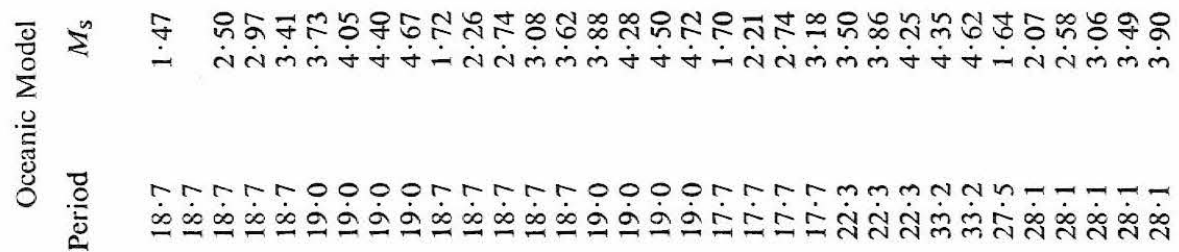

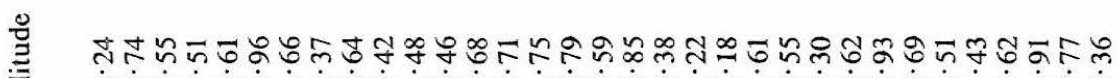

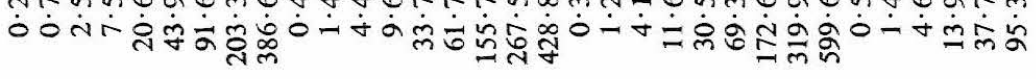

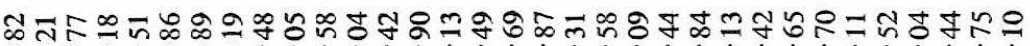

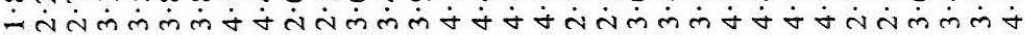

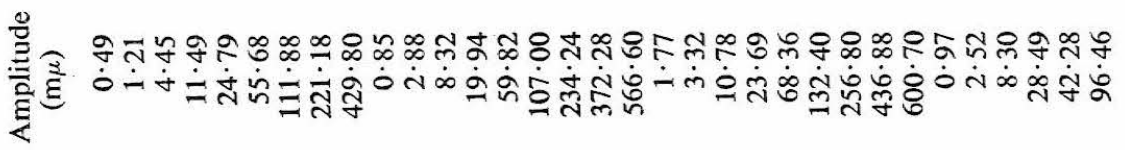

끌

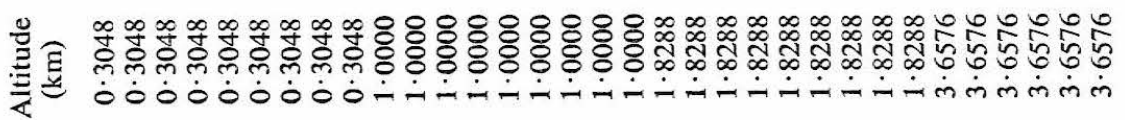




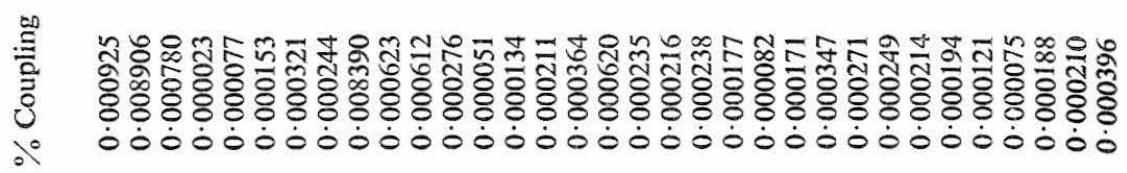

Ј

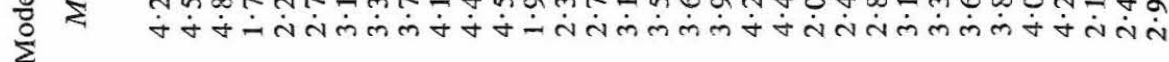

ฮूँ

7-75T.

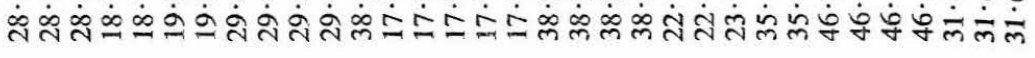

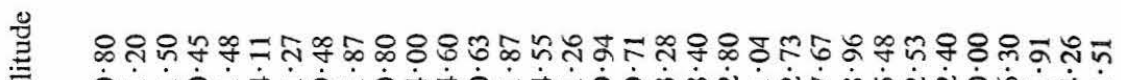

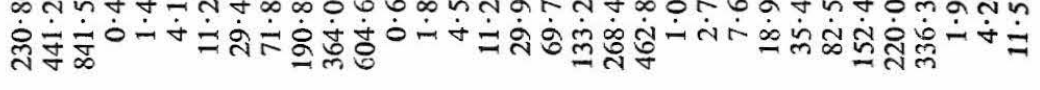

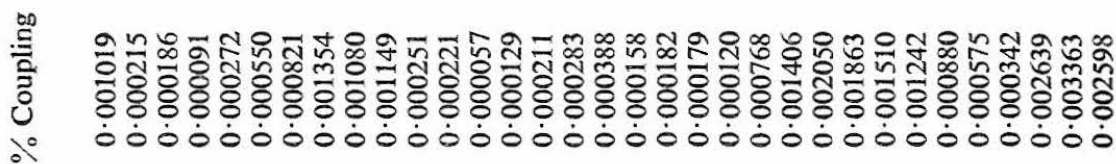

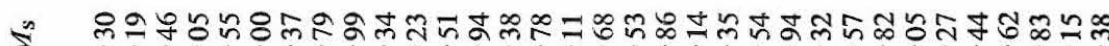

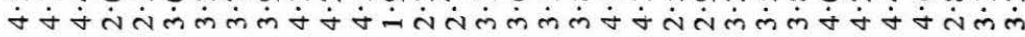

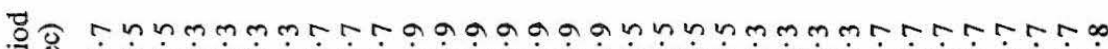
总鬼

节 ิ

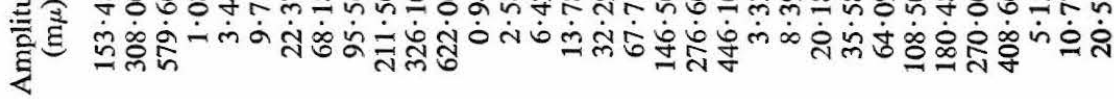

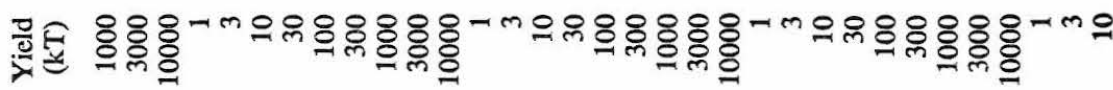

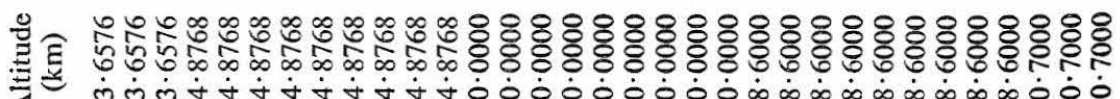

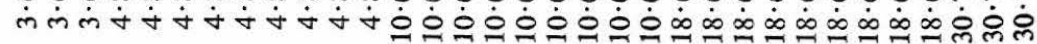




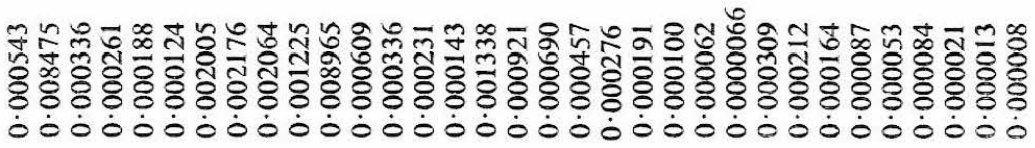

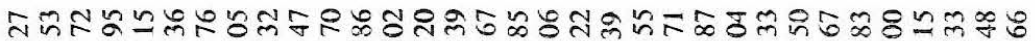

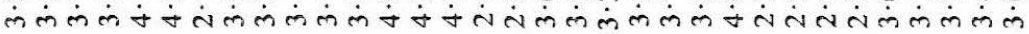

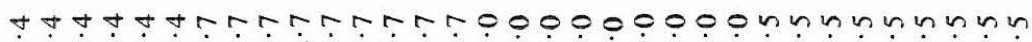

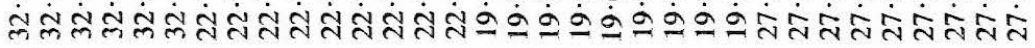

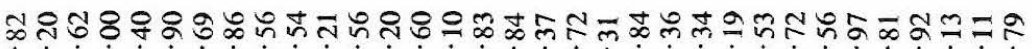

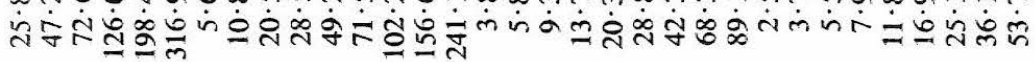

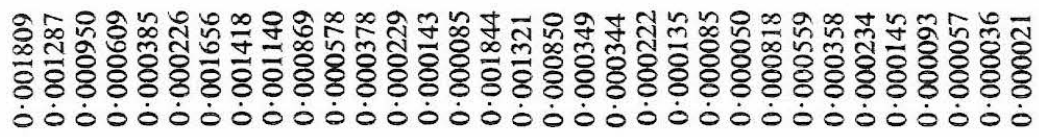

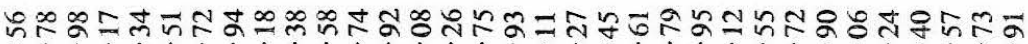

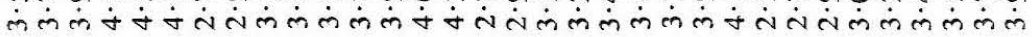

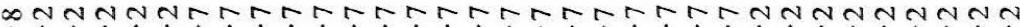

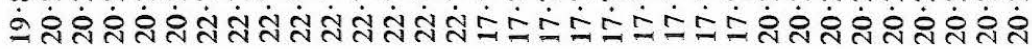

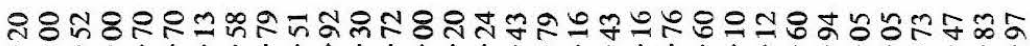

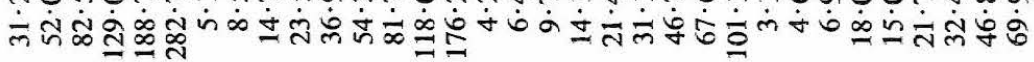

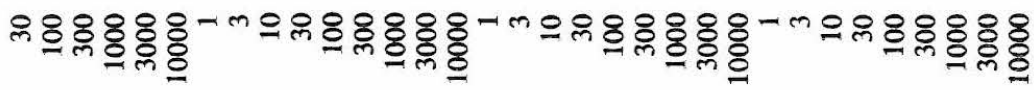

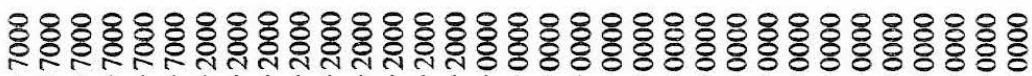

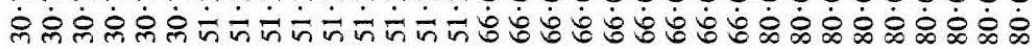


and burst altitudes to $80 \mathrm{~km}$. The seismic coupling, was also calculated. This is defined by the ratio of seismic energy to explosive yield, $E_{\mathrm{s}} / W$, where

and

$$
\log E_{\mathrm{s}}=9.4+2.14 M_{\mathrm{s}}-0.054 M_{\mathrm{s}}^{2} \quad \text { (Pomeroy 1963) }
$$

$$
M_{\mathrm{s}}=\log (A / T)+1.66 \log \Delta-0.18 .
$$

$A$ is peak-to-peak amplitude in $m \mu$ and $\Delta$ is epicentral distance in degrees. Fig. 12 shows the relation of $M_{\mathrm{s}}$ to yield and burst height for the continental and oceanic models. At most altitudes the continental $M_{\mathrm{s}}$ is greater than the oceanic $M_{\mathrm{s}}$ for a given yield; the exceptions are $10 \mathrm{~km}$ and $51.2 \mathrm{~km}$. At a burst height of $80 \mathrm{~km}$, both models show $M_{\mathrm{s}}$ proportional to $\log W^{\frac{1}{2}}$; and near $0 \mathrm{~km}, M_{\mathrm{s}}$ is proportional to $\log W$. Fig. 13 shows the relation of the maximum waveform amplitude $\left(A_{\mathrm{m}}\right)$ to yield and burst height for the two models. Except in the altitude range $40-60 \mathrm{~km}$, the maximum amplitudes for the continental model are greater than the oceanic model amplitudes. If a measured quantity could be found with contours intersecting the contours shown in Fig. 13 at angles approaching $90^{\circ}$, then we would be able to determine both yield
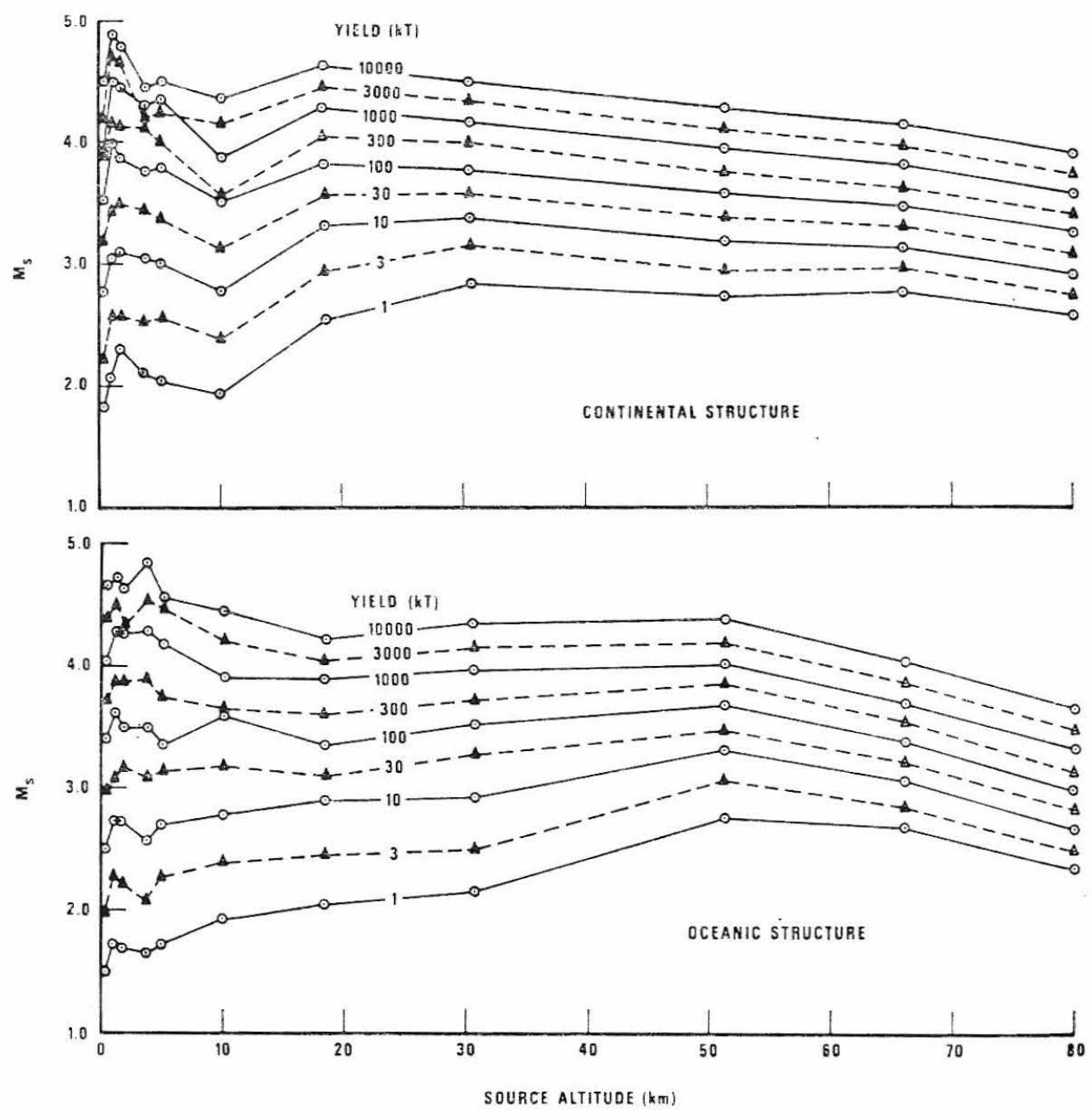

FIG. 12. $M_{\mathrm{S}}$ as a function of source altitude with yield as a parameter, for continental and oceanic structures. 


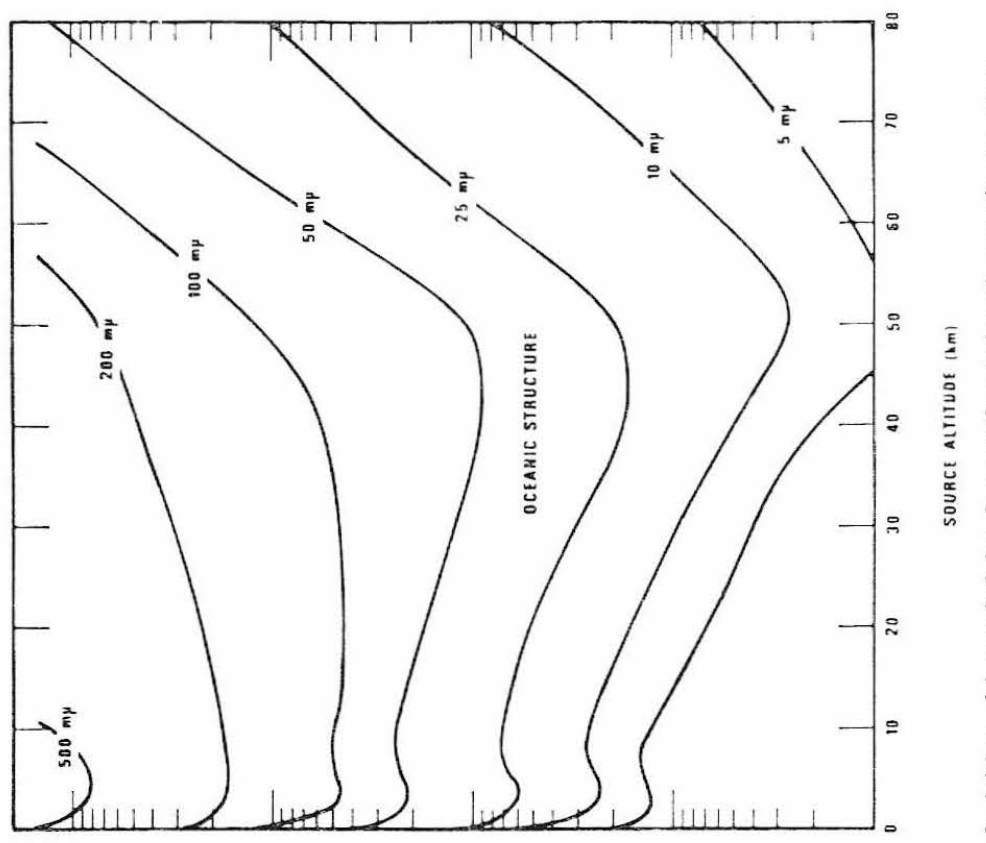

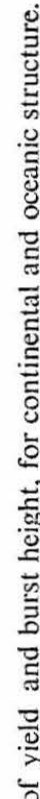

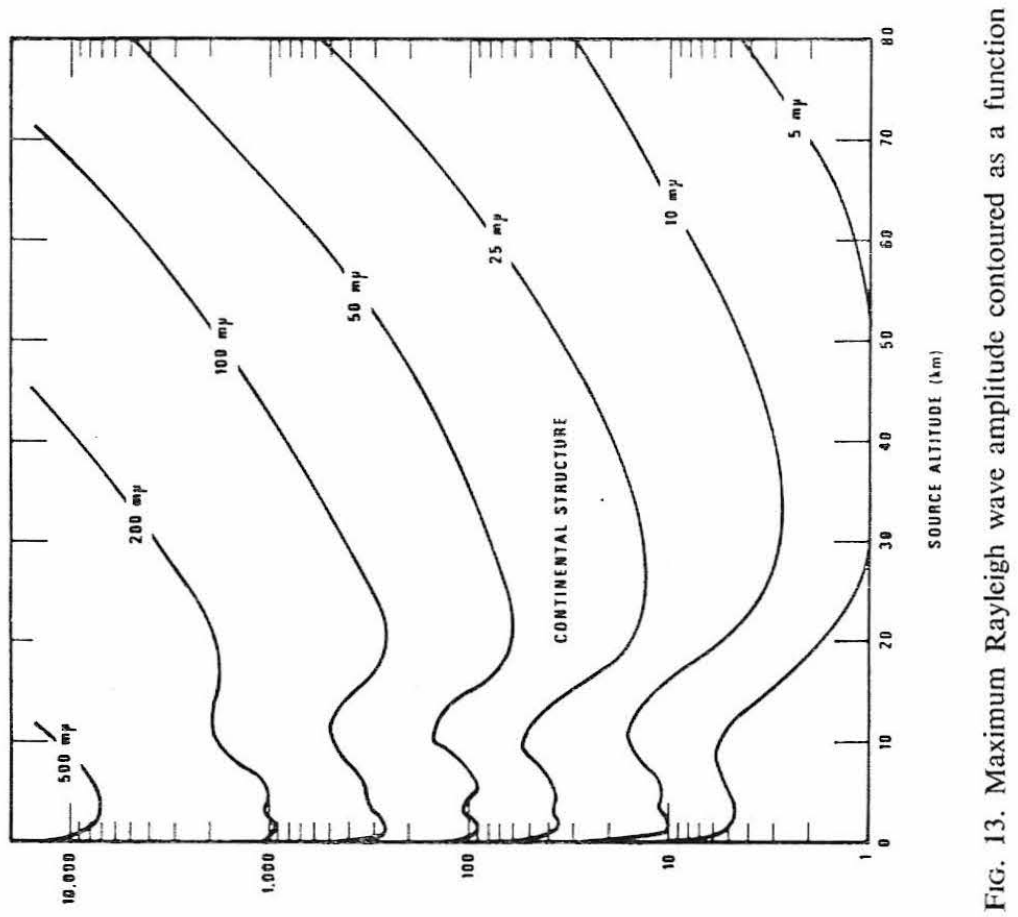

(14) 01314 


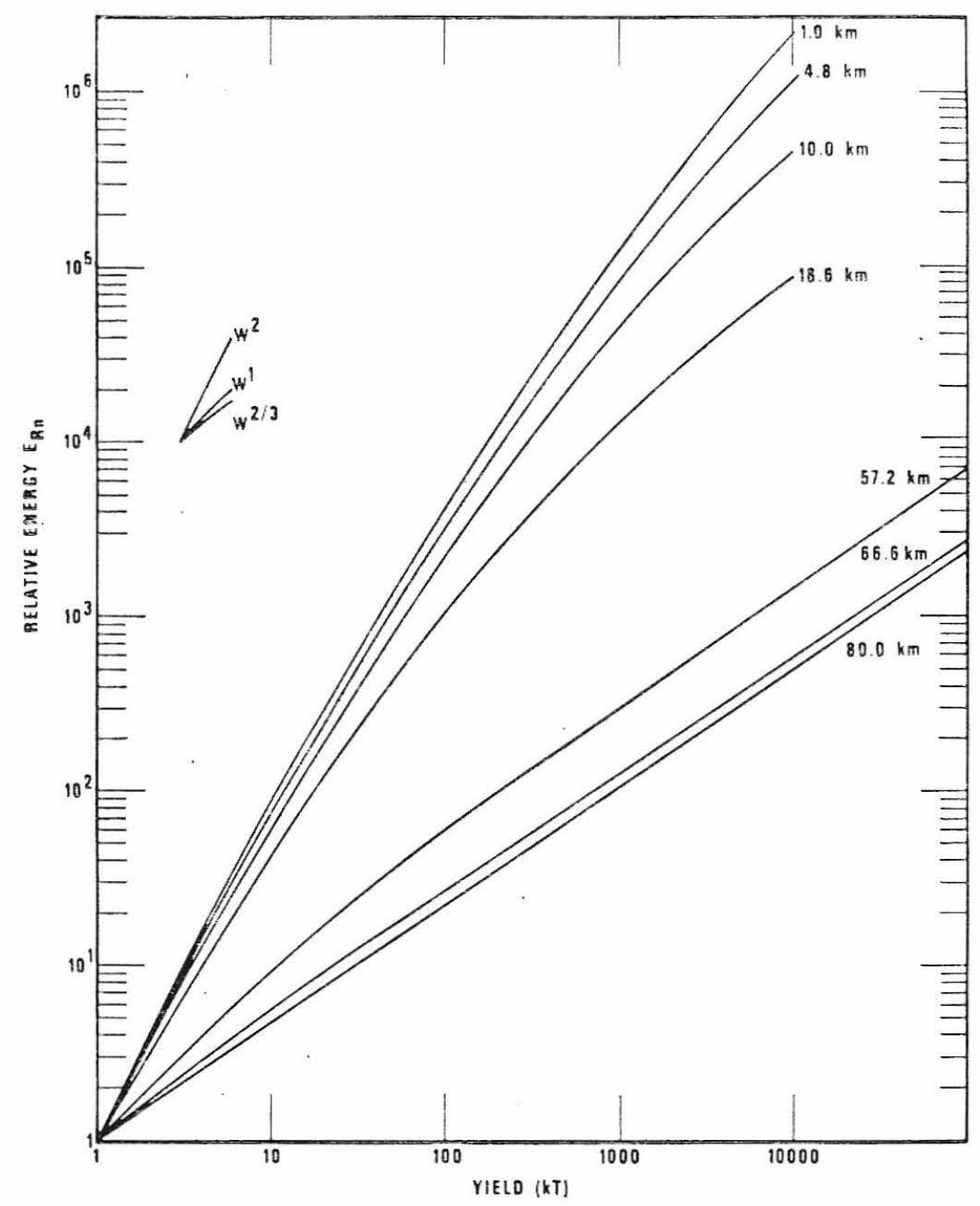

FIG. 14. Spectral energy (normalized at 20-s period) as a function of yield. Sources at altitudes 1.0 to $80.0 \mathrm{~km}$ over a continental Earth model. Reference lines show $W^{2}, W^{1}$, and $W^{3}$ yield dependence.

and burst height from the Rayleigh wave. Without such a quantity we could determine the yield if the burst height is known, but knowing only the yield, the burst height cannot be determined uniquely.

If the yield and burst height are known for one of a series of explosions at the same location, then the yields for all others detonated at approximately the same altitude can be calculated by comparing the energy spectra. Fig. 14 shows relative spectral energies, defined by

$$
E_{R_{N}}=\frac{1}{N} \sum_{i=1}^{N}\left(A_{i}{ }^{2} / A_{R_{i}}{ }^{2}\right)
$$

where $N$ is number of equally spaced spectral samples $A_{i}$, and the reference spectrum $A_{R}$ is calculated from the theoretical waveform for an explosion of $1 \mathrm{kT}$ at the same height and the same epicentral distance as the event with unknown yield. In terms of 
amplitudes rather than energies, the following conclusions can be drawn from Fig. 14:

1. Cube root scaling is observed for the amplitude spectra of Rayleigh waves from high-altitude explosions over continental paths.

2. For intermediate altitudes, the amplitude spectra have a $W^{1 / 2}$ dependence for low yields and $W^{1 / 3}$ for high yields.

3. For near-surface altitudes, the amplitude spectra have a $W$ dependence for high yields.

Further analysis revealed that when the amplitudes differ from cube root scaling, the shorter-period components are closer to the $W^{1 / 3}$ dependence than the longer-period components. The quantity $E_{R_{n}}$ was computed without smoothing the spectra $A$ and $A_{R}$, but the scatter in our results might be removed by smoothing at least $A_{R}$.

We examined the amplitude spectral minima and the phase spectra to see if there existed a simple relationship to the burst height, but none could be found.

Spectral splitting, i.e. the ratio of energy in the longer-period end of the Rayleigh
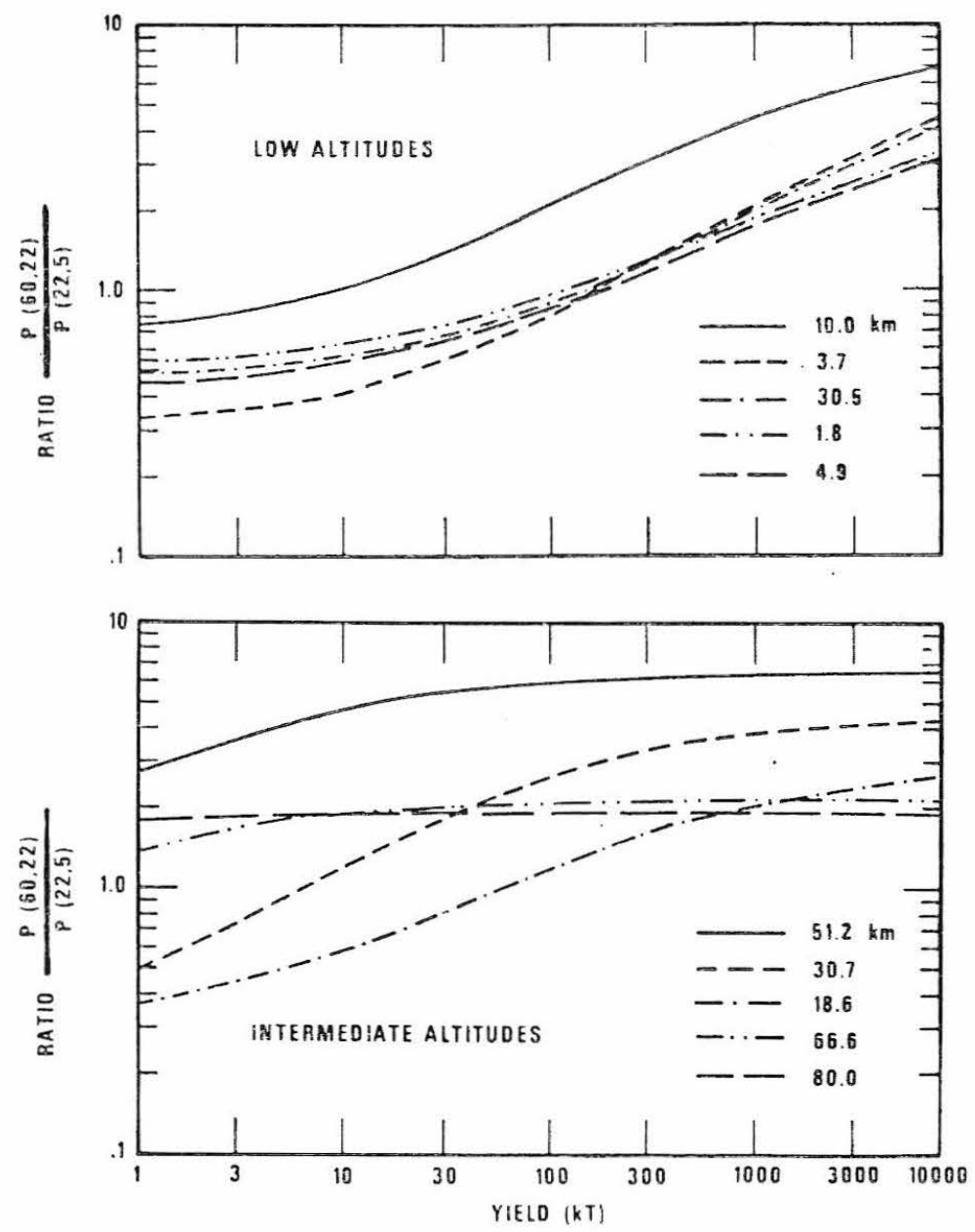

FIG. 15. Spectral power ratio $\langle R\rangle_{22}$ as a function of yield, for continental Earth structure. Upper figure for low source altitudes, upper for intermediate altitudes. 
wave spectrum to energy in the shorter-period end, has been suggested as a discriminant between underground explosions and earthquakes (von Seggern \& Lambert 1970). The physical rationale is that the two source types should be sufficiently different in nature that the shape of the radiated elastic wave spectrum should be measurably different. Intuitively it seems reasonable that variations in yield and burst height of atmospheric explosions might produce similar effects, so we studied the spectral distribution of energy in the theoretical Rayleigh wave calculations.

We define the power spectral splitting ratio by

$$
\langle R\rangle_{T}=\frac{P\left(T_{2}, T\right)}{P\left(T, T_{1}\right)}, \quad T_{1}<T \leqslant T_{2}
$$

where $P(a, b)=\sum_{i=a}^{b} A_{i}{ }^{2}$ and the $A_{i}$ are the Fourier amplitude coefficients. Von Seggern \& Lambert (1970) showed that the values of $\langle R\rangle_{T}$ obtained by splitting the spectra at $T=22 \mathrm{~s}$ separates the population of atmospheric explosion seismograms from earthquakes and underground explosion seismograms because the former contain less long-period energy than the latter. Putting $T_{1}=10 \mathrm{~s}$ and $T_{2}=62 \mathrm{~s}$, we obtain the values of $\langle R\rangle_{22}$ shown in Fig. 15 for the continental model. The only general relation we can conclude from these data is that for the higher burst altitudes there is a yield threshold above which $\langle R\rangle_{22}$ is constant. This relation is also observed

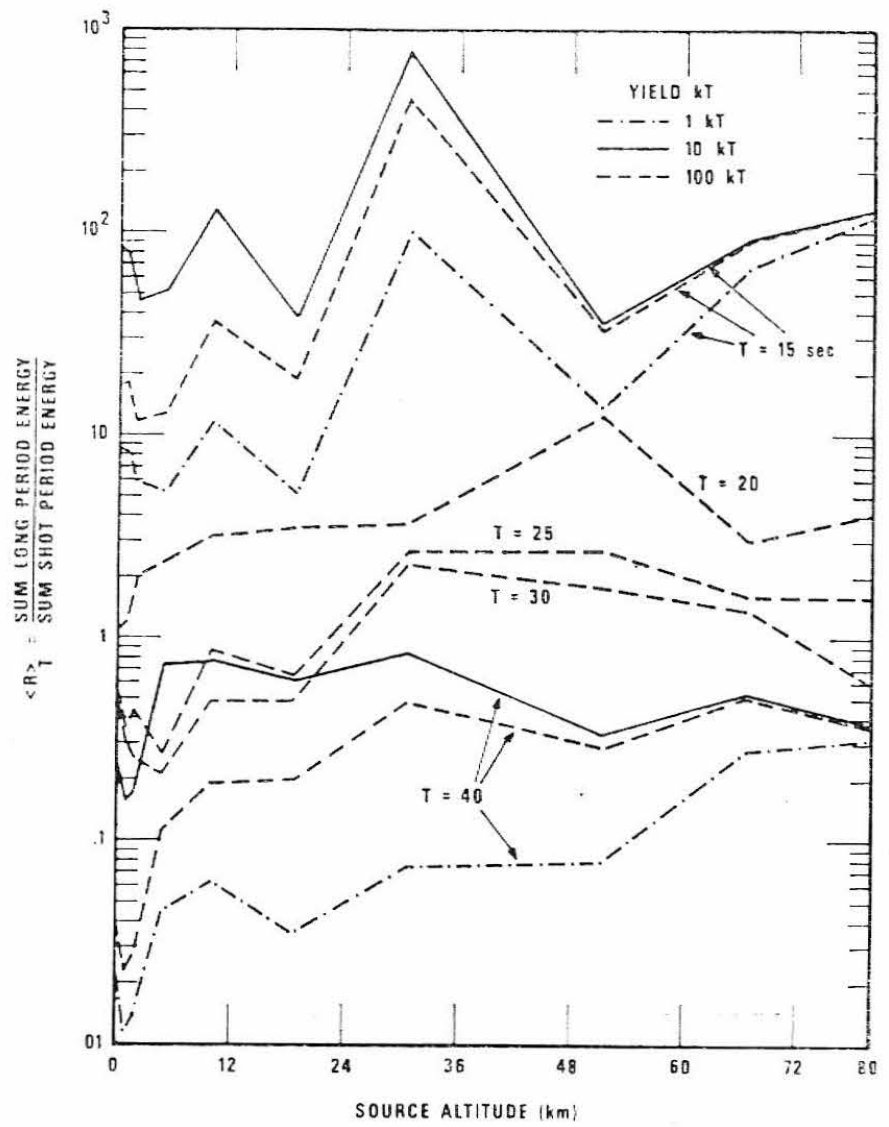

FIG. 16. Spectral power ratio $\langle R\rangle_{22}$ as a function of source altitude, for $T=15$ to $40 \mathrm{~s}$, and for three source yields. Continental Earth model. 
in Fig. 16, which gives $\langle R\rangle_{T}$ for $T=15,20,25,30$ and $40 \mathrm{~s}$ as a function of burst altitude for yields of 1,10 , and $10000 \mathrm{kT}$. In another attempt to use $\langle R\rangle_{T}$ for yield and burst height diagnostics, we calculated the function

$$
\widetilde{R}(T)=\log \left[T^{2}\langle R\rangle_{T}\right]
$$

and we found that the shapes of $\tilde{R}(T)$ were nearly independent of the yield but were distinguishable for different burst heights (Fig. 17). Unfortunately there does not appear to be any systematic variation that would permit a burst height prediction from $\widetilde{R}(T)$ when the source parameters are unknown.
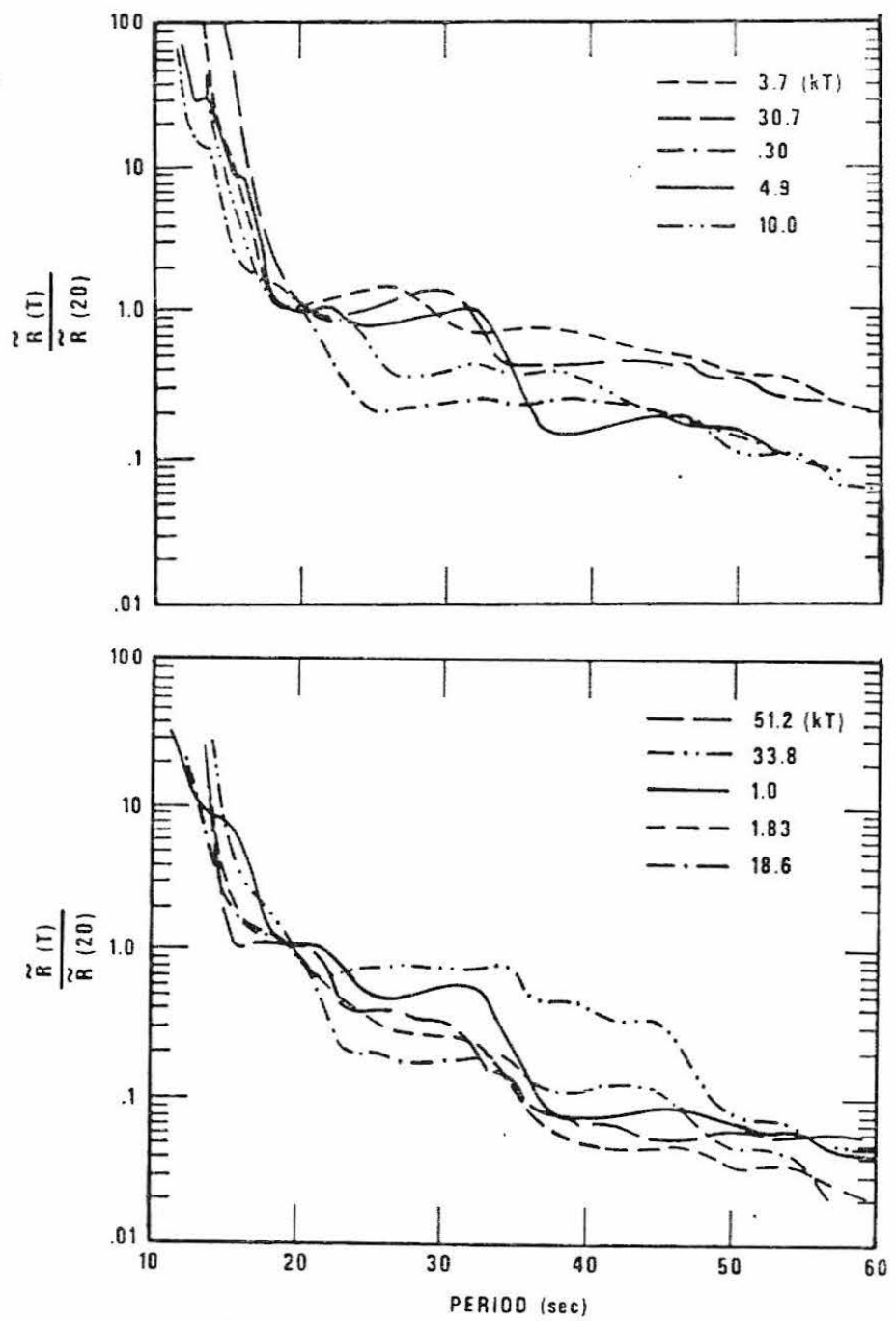

FIG. 17. $\widetilde{R}(T)$ normalized at $T=20 \mathrm{~s}$ period, for different source altitudes. The data are separated into two groups simply for clarity of presentation. 


\section{Conclusions}

Our theoretical treatment of Rayleigh waves generated by atmospheric nuclear explosions contains a number of approximations with regard to source spectra and scaling, the atmospheric model, and the Earth model. Improvements in these assumptions will require comparisons with observations from sources whose burst height and yield are known. The choice of peak overpressure scaling depends on such considerations.

Having analysed our theoretical data base to find source diagnostics, we found that source yield may be estimated if the source altitude is approximately known. We expect the variation of yield to result in a continuous change of the waveforms and spectra. In the case of source altitude we are dealing with higher modes of the atmosphere system due to the large Rayleigh phase velocities compared to the acoustic velocities of the atmosphere. This means there are many nodal points in the atmosphere eigenfunction with respect to altitude and frequency. Since this eigenfunction is a factor in the source altitude term (see equation (3)) the variations in the altitude resulted in nearly discontinuous changes in spectra. We conclude that the yield diagnostics may not be useful for determining source altitude.

The seismic coupling is generally greater for explosions over continents than over oceans; the difference may be as much as 0.5 magnitude units. The major differences resulting from using different Earth models are seen in the waveforms, which are governed largely by the group velocity dispersion. Since most travel paths are a combination of oceanic and continental segments, the effective dispersion will be a weighted average of the velocities used in computing these waveforms. The effect on magnitude measurements for mixed paths can be reduced by using path corrections derived from observed group velocity curves (Marshall \& Basham 1972). The real Earth paths will also cause changes in the amplitude spectra due to mode conversions and reflections where the structure is changing. In consideration of these effects (which we have not included in our analysis) we conclude that the best estimates of source altitude and yield will be made by comparing seismograms from explosions having common epicentres and travel paths, such as the technique used by Toksöz \& Ben-Menahem (1964).

\section{Acknowledgments}

We thank Allen D. Pierce of the Massachusetts Institute of Technology and Brian L. Murphy of Mt. Auburn Research Associates for useful discussions. This research was sponsored by the Advanced Research Projects Agency under Project Vela Hotel, and monitored by the Air Force Office of Scientific Research under contract F-14620-69-C-0082 with Teledyne Geotech and contract F-44620-70-C-0120 with the California Institute of Technology.

David G. Harkrider:

Seismological Laboratory, California Institute of Technology, Pasadena, California 91109
Carl A. Newton and Edward A. Flinn:

Alexandria Laboratories, Teledyne Geotech, Alexandria, Virginia 22314 


\section{References}

Aki, K., 1960. Study of earthquake mechanism by a method of phase equalization applied to Rayleigh and Love waves, J. geophys. Res., 65, 729-740.

Alewine, R. W., 1972. Theoretical and observed distance corrections for Rayleigh wave magnitude, Bull. seism. Soc. Am., 62, 1611-1619.

Ben-Menahem, A., 1965. Observed attenuation and $Q$ values of seismic surface waves in the upper mantle, J. geophys. Res., 70, 4641-4652.

Ben-Menahem, A. \& Harkrider, D. G., 1964. Radiation patterns of seismic surface waves from buried dipolar point sources in a flat stratified earth, J. geophys. Res., 69, 2605-2620.

Cagniard, L., 1962. Refection and refraction of progressive seismic waves, translated and revised by E. A. Flinn and C. H. Dix, McGraw-Hill Book Co., New York.

Dorman, J., 1962. Period equation for waves of Rayleigh type on a layered, liquidsolid half space, Bull. seism. Soc. Am., 52, 389-397.

Douglas, A., Hudson, J. A. \& Blamey, C., 1972. A quantitative evaluation of seismic signals of teleseismic distances-III, Computed $P$ and Rayleigh wave seismograms, Geophys. J. R. astr. Soc., 28, 385-410.

Gakenheimer, D. C., 1969. Response of an elastic half space to an expanding ring of surface pressure, Report RM-6095-PR., The Rand Corp., Santa Monica, California.

Gakenheimer, D. C., 1971. Response of an elastic half space to expanding surface load s, J. appl. Mechanics, 38, 99-110.

Gakenheimer, D. C. \& Miklowitz, J., 1969. Transient excitation of an elastic half space by a point load traveling on the surface, J. appl. Mechanics, 36, 505-515.

Glasstone, S., ed., 1957 and 1962. The effects of nuclear weapons, U.S. Government Printing Office, Washington, D.C.

Harkrider, D. G., 1964a. Theoretical and observed acoustic-gravity waves from explosive sources in the atmosphere, J. geophys. Res., 69, 5295-5321.

Harkrider, D. G., 1964b. Surface waves in multilayered elastic media, 1, Rayleigh and Love waves from buried sources in a multilayered elastic half-space, Bull. seism. Soc. Am., 54, 627-679.

Harkrider, D. G., 1970. Surface waves in multilayered elastic media, 2, Higher mode spectra and spectral ratios from point sources in plane layered earth models, Bull. seism. Soc. Am., 60, 1937-1987.

Harkrider, D. G. \& Anderson, D. L., 1966. Surface wave energy from point sources in plane layered earth models, J. geophys. Res., 71, 2967-2980.

Harkrider, D. G. \& Flinn, E. A., 1970. Effect of crustal structure on Rayleigh waves generated by atmospheric explosions, Rev. Geophys., 8, 501-516.

Hudson, J. A., 1969. A quantitative evaluation of seismic signals at teleseismic distances-I, Radiation from point sources, Geophys. J. R. astr. Soc., 18, 233-249.

Kerr, A. U., 1972. Digital computer programs for recording and processing infrasonic array data, Geophys. J. R. astr. Soc., 26, 21-40.

Lutsky, M. \& Lehto, D. L., 1968. Shock propagation in spherically symmetric exponential atmospheres, Phys. Fluids, 11, 1466-1472.

Marshall, P. D. \& Basham, P. W., 1972. Discrimination between earthquakes and underground explosions employing an improved $M_{\mathrm{s}}$ scale, Geophys. J. R. astr. Soc., 28, 431-458.

Murphy, Brian L., 1972. Variation of Rayleigh wave amplitude with yield and height of burst for intermediate-altitude nuclear detonations, J. geophys. Res., 77, 808-817.

Nickel, G. H. \& Whitaker, W. A., 1972. Distant seismic waves from a high-altitude source, Geophys. J. R. astr. Soc., 26, 369-378. 
Pierce, A. D., 1968. Theoretical source models for the generation of acoustic-gravity waves by nuclear explosions, in Acoustic-gravity waves in the atmosphere, ed. T. M. Georges, U.S. Government Printing Office, Washington, D.C.

Pierce, A. D., Posey, J. W. \& Iliff, E. F., 1971. Variation of nuclear explosion generated acoustic-gravity waveforms with burst height and with energy yield, J. geophys. Res., 76, 5025-5042.

Pomeroy, P. W., 1963. Long-period seismic waves from large near-surface nuclear explosions, Bull. seism. Soc. Am., 53, 109-150.

Press, F. \& Harkrider, D. G., 1962. Propagation of acoustic-gravity waves in the atmosphere, J. geophys. Res., 67, 3889-3908.

Reed, S. G., 1959. Note on finite amplitude propagation effects on shock wave travel times from explosions at high altitude, J. acoust. Soc. Am., 31, 1265.

Toksöz, M. N. \& Ben-Menahem, A., 1964. Excitation of seismic surface waves by atmospheric nuclear explosions, J. geophys. Res., 69, 1639-1648.

von Seggern, D. \& Lambert, D. G., 1970. Theoretical and observed Rayleigh-wave spectra for explosions and earthquakes, J. geophys. Res., 75, 1382-1402.

Wares, G. W., Champion, K. W., Pond, H. L. \& Cole, A. E., 1960. Model atmosphere in Handbook of geophysics, 1-1, 1-37, The MacMillan Co., New York.

\section{Appendix A}

\section{Disc pressure source}

Our assumed pressure source is constant over a disc of radius $a$, and with a transformed time history $B(\omega)$, i.e.

$$
\left\langle p_{\mathbf{s}}(r, \theta)\right\rangle= \begin{cases}B(\omega), & r<a \\ 0, & r>a .\end{cases}
$$

Using the area integration technique, we obtain

$$
\begin{gathered}
\langle w(r, \theta)\rangle=B(\omega) \int_{0}^{2 \pi} \int_{0}^{a} G\left(\mathbf{r} \mid \mathbf{r}_{0}\right) r_{0} d r_{0} d \theta_{0} \\
=\frac{-i}{2 \pi} B(\omega) \int_{0}^{\infty} \frac{[G N-L H]}{F_{\mathrm{R}}} \int_{0}^{2 \pi} \int_{0}^{a} J_{0}(k R) r_{0} d r_{0} d \theta_{0} d k
\end{gathered}
$$

where

$$
R^{2}=r_{0}^{2}+r^{2}-2 r r_{0} \cos \left(\theta-\theta_{0}\right) \text {. }
$$

From the addition theorem of Bessel functions,

$$
J_{0}(k R)=\sum_{m=0}^{\infty} \varepsilon_{m} J_{m}\left(k r_{0}\right) J_{m}(k r) \cos \left[m\left(\theta-\theta_{0}\right)\right]
$$

where the Neumann factor $\varepsilon_{m}$ is defined as

Since

$$
\varepsilon_{m}= \begin{cases}1, & m=0 \\ 2, & m \neq 0 .\end{cases}
$$

$$
\cos \left[m\left(\theta-\theta_{0}\right)\right]=\cos (m \theta) \cos \left(m \theta_{0}\right)-\sin (m \theta) \sin \left(m \theta_{0}\right)
$$


the integration over $\theta$ yields

$$
\langle w(r, \theta)\rangle=-i B(\omega) \int_{0}^{\infty} \frac{[G N-L H]}{F_{\mathbf{R}}} J_{0}(k r) \int_{0}^{a} J_{0}\left(k r_{0}\right) r_{0} d r_{0} d k
$$

where we have used the relations

and

$$
\int_{0}^{2 \pi} \cos (m \theta) d \theta= \begin{cases}0, & m \neq 0 \\ 2 \pi, & m=0\end{cases}
$$

$$
\int_{0}^{2 \pi} \sin (m \theta) d \theta=0 \text { for all } m
$$

Since

$$
\int_{0}^{a} J_{0}\left(k r_{0}\right) r_{0} d r_{0}=\frac{a}{k} J_{1}(k a)
$$

our solution for the disc source is:

$$
\left\langle w_{0}(r, \theta)\right\rangle=-i B(\omega) a \int_{0}^{\infty} \frac{[G N-L H]}{F_{\mathrm{R}}} \frac{J_{1}(k a)}{k} J_{0}(k r) d k .
$$

This solution can be verified using equations (4) and (9). The disc source can be expressed in integral form as

$$
\left\langle p_{s}(r, \theta)\right\rangle=B(\omega) a \int_{0}^{\infty} J_{1}(k a) J_{0}(k r) d k
$$

and comparison with equation (4) implies that

$$
P_{\mathrm{s}}=B(\omega) a J_{1}(k a)
$$

which substituted in equation (9) gives the same result as equation (A2). 